\title{
Elisa Brilli Enrico VII, Dante e gli «universaliter omnes
Tusci qui pacem desiderant»
}

Destinatari e (co-)mittenti danteschi

\begin{abstract}
Nell'intestazione della VII epistola a Enrico VII del 17 aprile 1311, Dante si accredita quale primo mittente del testo, ricorrendo alla dicitura di «exul inmeritus», già consueta nella sua precedente produzione epistolografica, ma aggiunge il riferimento, quali secondi mittenti del testo, agli «universaliter omnes Tusci qui pacem desiderant». Ciò contrasta con il ritratto che sarà tracciato da Cacciaguida e con la sua affermazione che, a partire dal 1304 circa, Dante avrebbe fatto «parte per sé stesso» (Par. XVII, 69). Mancando peraltro attestazioni documentarie di un qualche incarico ufficiale ricoperto da Dante in questa fase politica, gli studiosi appaiono divisi tra chi immagina un'allusione ai fuoriusciti di parte bianca e ghibellina e chi pensa piuttosto che il riferimento sia ai conti Guidi favorevoli al Lussemburghese. Attraverso l'esame della totalità della documentazione storica e letteraria superstite, l'articolo scarta entrambe tali ipotesi e suggerisce invece di riconoscere in questo enigmatico riferimento la traccia del tentativo di Dante di creare una nuova comunità in sostegno di Enrico; un tentativo che, in una prospettiva di lunga durata, costituisce l'ideale punto di transizione dalle modalità di costruzione del pubblico già proprie all'autore della Vita nova a quelle che contraddistingueranno l'attitudine profetica dell'autore delle ultime due cantiche della Commedia. L'articolo offre inoltre una messa a punto ecdotica a proposito dell'intitulatio, archiviando la proposta ricostruttiva di E. Pistelli, passata in giudicato in tutte le edizioni posteriori.
\end{abstract}

In the superscription of his epistle VII to Henry VII (April 17, 1311), Dante validates himself as the first sender through the clause «exul inmeritus», already in use in his previous letters; he also refers to «universaliter omnes Tusci qui pacem desiderant» as second senders of the epistle. This is in conflict with the biographical portrait that will be presented by Cacciaguida, and especially with the claim that, from 1304 onwards, Dante's party would have been himself (Par. XVII, 69). Since we lack documents witnessing some official charge entrusted to Dante in this political phase, scholars are divided between those who read the clause as referring to White and Ghibelline exiles, and those who see

Elisa Brilli, University of Toronto

Ә Open Access. () 2020 Elisa Brilli, published by De Gruyter. (c) BY This work is licensed under the Creative Commons Attribution 4.0 International License (CC BY 4.0).

https://doi.org/10.1515/9783110590661-019 
in it a reference to the Guidi Counts, who supported Henry VII. By examining all surviving historic and literary documents, I dismiss both readings and propose to read this enigmatic reference as a witness of Dante's attempt to create a new community in support of Henry. An attempt that, in a long-term perspective, represents an ideal moment transition in the development of Dante's strategies for shaping his audience, from those employed in his Vita nova to those which mark the prophetical attitude of the Purgatorio and Paradiso. The article also provides a new textual reading for the letter's superscription, thus dismissing Pistelli's proposal, who had been accepted in all subsequent editions.

Parole chiave: Dante Alighieri, Performatività, Profetismo, Salutatio, Epistolografia.

Questo contributo si concentra sulla salutatio (o intitulatio in termini retorici, o ancora protocollo in termini diplomatici) della VII epistola, la terza della stagione arrighiana, indirizzata all'imperatore il 17 aprile 1311. Mi soffermerò su questa porzione testuale per riconsiderare, in sede sia storica che retorica, le interpretazioni che ne sono state offerte negli studi. Questo esame, pur se focalizzato su un dettaglio in apparenza secondario della produzione epistolografica dantesca, vorrebbe contribuire a rivalutare l'intero iter del Dante epistolografo tra il settembre del 1310 e l'aprile del 1311, e in particolare l'annosa questione dell'attitudine, o habitus, profetico adottato (o meno) dal redattore di questi testi e che tanta parte ha nella produzione letteraria coeva e posteriore del fiorentino.

\section{Chiose testuali}

Rileggiamo innanzitutto la salutatio della VII epistola così come accolta dalle edizioni a partire da quella curata da Ermenegildo Pistelli nel 1921:

Sanctissimo gloriosissimo atque felicissimo triumphatori et domino singulari domino Henrico divina providentia Romanorum Regi et semper Augusto, devotissimi sui Dantes Alagherii Florentinus et exul inmeritus ac universaliter omnes Tusci qui pacem desiderant, terre osculum ante pedes.

Come informano tutti i commenti, l'appellativo riservato a Enrico «divina providentia Romanorum Rex et semper Augustus» è affatto tradizionale, prima s'intende dell'incoronazione romana. ${ }^{1}$ Tuttavia, è stato di recente notato che il pri-

1 Conforme cioè alla sottoscrizione degli atti ufficiali fino all'incoronazione a Roma (29 giugno 1312) quando, come consueto, il titolo è aggiornato in quello di «imperator» (come ricorda 
mo degli attributi riferiti all'imperatore, il «sanctissimus», rompe il protocollo epistolografico trasponendo all'autorità imperiale un aggettivo tradizionalmente riservato alla pontificia in varie artes dictaminis e attestazioni. ${ }^{2}$ Data la delicatezza della faccenda poiché, come non serve ricordare, la salutatio è la parte retoricamente più importante di un'epistola, conviene riconsiderare quel che è trasmesso dai codici. Li elenco qui di seguito in ordine cronologico, riproducendo il testo secondo le edizioni diplomatiche approntate da P. Toynbee e F. Mazzoni, nuovamente verificate sulle riproduzioni dei manoscritti, nonché le edizioni dei volgarizzamenti approntate da A. Montefusco:

Roma, Biblioteca Nazionale Centrale, Fondo S. Pantaleo 8 (101) (P), fol. 141r: Gloriosissimo atque felicissimo triumphatori et domino singulari domino Henricho divina providentia Romanorum Regi et senper Augusto devotissimj suj Dantes alagherii florentinus et exul inmeritus ac universaliter omnes Tuscj quj pacem desciderant terre obsculum ante pedes. $^{3}$

Città del Vaticano, BAV, Pal. Lat. $1729(\mathrm{~V})$, fol. 56r dove l'epistola, anepigrafa, è introdotta dalla rubrica: Epistola Dantis Alegerii florentini ad henricum cesarem augustum. ${ }^{4}$

Siena, Biblioteca Comunale degli Intronati, F.V.9 (S), fol. 124r: Gloriosissimo ac felicissimo triumphatori domino singulari domino Henrico divina providentia Romanorum Regi semper augusto, Devotissimi tui Dantes Aldigherii Florentinus et exul inmeritus ac universaliter omnes tusci qui pacem desiderant, terre osculum ante pedes. ${ }^{5}$

Venezia, Biblioteca Nazionale Marciana, Lat. XIV 115 (4710) (M), fol. 8r: Sanctissimo Triumphatori et domino singulari Domino Henrico divina providentia Romanorum rege

anche Fenzi, Ancora a proposito, p. 99). La stessa formula è adottata da un altro fiorentino per rivolgersi all'imperatore: Francesco da Barberino che scrive a Enrico VII a nome della Corona Romana tra il giugno e il dicembre del 1311 per spronarlo ad accelerare l'incoronazione («excellentissimo ac serenissimo principi et domino suo, domino Henrico, divina favente clementia Romanorum regi dignissimo et semper augusto», ed. in Brilli, Fontes, Montefusco, Sedurre l'imperatore, p. 76), ma appunto se ne trovano innumerevoli casi essendo corrente.

2 Così ad es. nei commenti Ep. (Pastore Stocchi), pp. 54-55; Ep. (Villa), p. 1551; Ep. (Baglio), pp. 20 e 155 (pur con giusta cautela, cfr. infra).

3 Cfr. la fotoriproduzione in Ep. (Witte); la trascrizione diplomatica in Toynbee, The S. Pantaleo Text, p. 209, da integrare con la descrizione, inclusiva della bibliografia pregressa, di Montefusco, Le "Epistole", pp. 442-445.

4 La medesima rubrica è appuntata nell'angolo in alto a destra dello stesso fol. forse da Francesco Piendibeni da Montepulciano, copista del manoscritto secondo Billanovich, Giovanni del Virgilio, pp. 211-213 e Mazzoni, Le epistole, p. 89, ma si vedano le cautele di Rossetto, Per il testo, p. 73 e di Baglio in Ep. (Baglio), p. 29. La riproduzione fotografica della sezione dantesca di questo codice edita da Schneider, Dantis Alagherii Monarchiae Liber, è ormai rimpiazzata dalla riproduzione integrale accessibile on-line su $<$ https://digi.vatlib.it $>$.

5 Trascrizione diplomatica di Mazzoni, Il Codice S(enese), p. 686. Non riverificato sul manoscritto. 
semper augusto devotissimi sui Dantes Aldigherrj florentinus et exul immeritus ac universaliter omnes Thusci qui pacem desiderant terre obsculum ante pedes. ${ }^{6}$

La salutatio è inoltre trasmessa dal volgarizzamento A:

Al gloriosissimo e felicissimo trionfatore, e singulare signore, Messere Arrigo, per la divina providenzia Re de' Romani et sempre accrescitore, e suoi devotissimi Dante Aleghieri fiorentino et sbandito immeritamente, e universalmente tutti e toscani che pace desiderano, a.llui alla terra dinanzi a' piedi baci mandano. ${ }^{7}$

e dal più fortunato volgarizzamento $\mathrm{B}$ :

Al gloriosissimo e felicissimo trionfatore, e singolare signore Messer Arrigo, per la divina provvidenza Re de’ Romani e sempre accrescitore, i suoi devotissimi Dante Alighieri, Fiorentino e non meritevolmente sbandito, e tutti i Toscani universalmente, che pace desiderano, mandano baci alla terra dinanzi a' vostri piedi. ${ }^{8}$

In assenza di $V$, la lezione «sanctissimo t.» è solo del tardo $M$ (post 1480), che la dà in luogo del «gloriosissimo atque $[\mathrm{ac} S]^{9}$ felicissimo t.» di $P$ e $S$, così come del «al gloriosissimo e felicissimo t.» di entrambi i volgarizzamenti. Come e perché si è giunti al trittico «sanctissimo gloriosissimo atque felicissimo t.»? Le edizioni ottocentesche e dei primi anni del Novecento, essendo approntate su $M$ come quella di Witte del 1827 (da cui in parte dipendono), leggono tutte «sanctissimo t.». ${ }^{10} \mathrm{Nel} 1912$ tuttavia Paget Toynbee diede alle stampe la trascrizione diplomatica dell'epistola secondo $P$, un codice già segnalato dalla Bibliografia dantesca di Paul Colomb de Batines e poi da Michele Barbi; quindi, prima in un articolo e poi nell'edizione oxoniense del 1920, corresse il textus receptus editando, con $P$, «gloriosissimo atque felicissimo t.» e segnalando in apparato la diversa lezione di $M$ e delle edizioni anteriori. ${ }^{11}$ La correzione di Toynbee fu

\footnotetext{
6 Cfr. la fotoriproduzione in Ep. (Witte) e la trascrizione diplomatica in Toynbee, The Venetian Text, p. 434.

7 Testo edito in Volgarizzamento (Montefusco), p. 271, cui rinvio per la tradizione manoscritta.

8 Volgarizzamento (Montefusco), p. 275.

9 Mazzoni, Il codice S(enese), p. 689 annoverava illvogo come esempio di «un intento correttorio talora di stampo umanistico».

10 Cfr. Ep. (Witte), p. 30; Ep. (Torri), p. 52; Ep. (Fraticelli), p. 488; Ep. (Fraticelli²), p. 464 (e varie riedizioni); Ep. (Giuliani), p. 22; Ep. (Moore), p. 409 (e così le seguenti).

11 Toynbee, The S. Pantaleo Text e quindi Ep. (Toynbee), p. 87 (già anticipato dall'ed. critica Toynbee, Dante's letter to the Emperor), e cfr. le segnalazioni di Colomb de Batines, Bibliografia dantesca, t. II, p. 209, e Barbi, rec. a Scartazzini, p. 23. Su $P$ si vedano ora le integrazioni offerte da Montefusco, Le “Epistole”, pp. 443-444. Ricordo che all'epoca dell'ed. Toynbee era ancora ignoto $S$ (segnalato solo nel 1939 da Mancini, Un nuovo manoscritto) che conferma la lezione adottata dall'editore inglese.
} 
accolta dall'edizione a cura di Monti del 1921 (p. 180), ma non convinse Ermenegildo Pistelli al quale spetta la paternità del testo oggi vulgato, ossia di un ircocervo frutto della fusione delle lezioni di $M$ e di $P$. Nell'edizione dell'epistola pubblicata in appendice alla scolastica Piccola antologia della Bibbia Volgata nel 1915, Pistelli editava la salutatio con la terna di attributi confessando «non so rinunziare al sanctissimo, che esce dal solito formulario ed è più dantesco», e la medesima soluzione fu adottata nell'edizione nazionale del $1921 .{ }^{12}$ La scelta e spiegazione di Pistelli furono brevemente chiosate da Ernesto Giacomo Parodi senza prendere posizione, almeno apertamente. ${ }^{13}$ Dall'ultradantismo di Pistelli e dal possibilismo di Parodi, via l'edizione nazionale, la salutatio con triplice attributo si è trasmessa fino alle edizioni più recenti inerzialmente e dando persino luogo a qualche grattacapo, come se non si sapesse più da dove fosse saltata fuori. ${ }^{14}$

12 Cfr. Piccola antologia della Bibbia volgata, pp. 209-219, cit. a p. 210; peraltro, in questa nota, Pistelli confondeva l'assetto testimoniale attribuendo a $M$ la lezione di $P$ e viceversa (ringrazio A. Montefusco per avermi procurato le riproduzioni di questa edizione); e la ristampa del 1919, stereotipa, conserva l'errore (sempre a p. 210). Si veda inoltre Ep. (Pistelli) p. 426, dove tuttavia mancano glosse (né qualcosa si trova a riguardo nelle righe consacrate alle epistole nella prefazione di M. Barbi, alle pp. XIX-XX). La lezione non rientra tra quelle discusse nel saggio preparatorio di Pistelli, Dubbi e proposte. Più in generale, si ricorderà la diffidenza, se non proprio insofferenza, di Pistelli per le minuzie filologiche, ben testimoniata dalle osservazioni svolte in veste di recensore di Toynbee: «come pretendere che ogni lettore debba farsi, per dir così, un'edizione critica per conto suo, vagliando da sé elementi di troppo diverso valore che gli sono offerti dall'apparatus? Osservazione che io credo non inutile, perché abbondando, pur troppo, gli apparatus di questo genere, che di chiamarsi critici non hanno davvero diritto» (Pistelli, rec. a Toynbee, p. 58, circa la scelta di registrare errori, alcuni dei quali delle precedenti ed.), nonché il fatto che Pistelli non lavorò con metodo filologico, a causa anche della difficile congiuntura storica, come ricorda Mazzoni, Riflessioni sul testo, p. 436 (p. 291 dell'anastatica).

13 Parodi, Intorno al testo, p. 142: «piuttosto voglio raccogliere qui una proposta del Pistelli, che riguarda proprio le prime parole del titolo. Come si sa, dei due codici che lo conservano, Pant. ${ }^{1}[=P]$ ha Gloriosissimo atque felicissimo Triumphatori (insieme con Pant. ${ }^{2}$ e l'altra versione [i due volgarizzamenti], che è adottato dal Toynbee; Ven. [= $M]$, seguito dal Moore ecc., ha Sanctissimo tr. Il Pistelli invece di scegliere, combina: Sanctissimo, gloriosissimo atque felicissimo tr. Quel Sanctissimo gli pare troppo dantesco perché si possa rinunziarci, ed è ben possibile ch'egli abbia ragione». Dato che Parodi corregge silenziosamente la recensio pasticciata di Pistelli (cfr. supra nota 12), non è improbabile che l'osservazione sia da prendere in senso velatamente polemico.

14 Adottano (e/o traducono) la soluzione approntata da Pistelli: Ep. (Vinay), p. 300; Ep. (Del Monte), p. 771-772; Ep. (Pézard), pp. 767; Ep. (Brugnoli-Frugoni), p. 562; Ep. (Jacomuzzi), p. 400; Ep. (Lokaj), p. 630; Ep. (Honess), pp. 71; Ep. (Villa), p. 1464. L'imporsi del testo del 1921 ha poi talvolta occasionato l'attribuzione della lezione supposta integra (ossia con i tre aggettivi, che come si è visto come tale non è attestata) ora a questo ora a quel testimone, procedendo 
Per dare un altro infimo contributo all'accidentata storia editoriale delle epistole, nonostante le validissime cure prodigate negli ultimi anni, la fusione delle due lezioni dell'edizione Pistelli, una fusione che può ben classificarsi come un altro esempio tanto della «fedeltà ancora eccessiva alla vulgata Fraticelli-Witte» quanto dell' «abuso di iudicium - non di rado mal fondato - che ha prodotto un testo critico caratterizzato da una vera e propria "furia" emendativa», ${ }^{15}$ pare da archiviarsi. Ciò in ragione di a) la minore plausibilità di una doppia omissione (di «sanctissimo» in $P, S$, volg. A, volg. B, e di «gloriosissimo et felicissimo» in $M$ ) rispetto a sola corruttela, nonché di b) le complicazioni che l'ipotesi di una doppia omissione di tal fatta, di valore separativo, implicherebbe sui rapporti tra i testimoni quali sono stati in seguito chiariti da Francesco Mazzoni; ${ }^{16}$ e c) il raffronto con l'aggettivazione riservata all'imperatrice Margherita nella salutatio dell'epistola VIII - «gloriosissime atque clementissime» così come delle variationes nella IX - «serenissime atque piissime» - e nella X «illustrissime atque piissime»-, non tanto perché non vi figura nessun «san-

per esclusione. Lo stesso Mazzoni affermò che $M$ presentasse la triplice attribuzione, e suggerì la caduta di «Sanctissimo» in $P$ e $S$ fosse «frutto di poligenesi (per saut du même au même)» (Mazzoni, Il codice S(enese), p. 690; rist. anast. p. 285), spiegazione però impervia data la posizione incipitaria. In Ep. (Padoan), p. 53 si afferma che «la salutatio integra si legge in $P$, mentre $V$ reca soltanto la didascalia» senza discutere le lezioni dei codd. Il commento Ep. (Baglio), p. 155 attribuisce la «formula incipitaria completa» a $P$ e imputa l'omissione del «Sanctissimo» a $M$ (senza rif. a $S$ e ai volgarizzamenti): aldilà della svista circa l'assetto testimoniale, è comunque condivisibile la riserva nei confronti dell'autenticità del reperto promosso a testo. In concomitanza con l'elaborazione del presente lavoro, ha messo in dubbio la sensatezza della soluzione vulgata Montefusco in un intervento orale, ora edito, che lo ringrazio per aver condiviso con me in una versione preliminare: Montefusco, Ancora su epistole, pp. 24-28.

15 Montefusco, Le "Epistole”, p. 412. Si può ben parlare qui di «fedeltà» al textus receptus Witte-Fraticelli poiché, come già notato, è essenzialmente dall'adesione ad esso che derivava la pena di Pistelli nel disfarsi della lezione di M, e di una certa, sebbene minore che in altri casi, «furia emendativa» nel supporre che tutta la tradizione manoscritta fosse in questo punto corrotta e latrice solo di membra disiecta della salutatio dantesca.

16 Dato che, per quest'epistola, «la tradizione si organizza in due famiglie $\alpha$ e $\beta$ provenienti da un comune archetipo; che l'unico rappresentate di $\alpha$ è l'autorevole $V(. .$.$) ; che la seconda$ famiglia si suddivide in due sottogruppi $x$ e $y$, al primo dei quali appartiene il codice $P$ unitamente alla seconda parte del mutilo $S$, mentre a $y$ appartiene il codice $M$, unitamente alla prima parte di $S$, che evidentemente, in quando individuo, deve aver tenuto presenti due diversi antigrafi della medesima tradizione $\beta »$ (Mazzoni, Le epistole, pp. 76-77 [pp. 256-257 dell'anast.]; si vedano anche i dettagli forniti da Mazzoni, Il codice S(enese), pur con l'avvertenza di cui alla nota precedente, e la sintesi in Mazzoni, Riflessioni sul testo, pp. 436-437 [pp. 291-292 dell'anast.]). Si sarebbe dunque costretti a ipotizzare che $S$, avendo sotto gli occhi una lezione di $y$ o con la terna d'attributi o già limitata al solo «Sanctissimo» come in $M$, si risolvesse a trascrivere la lezione di $P$ che però rispetto a quella del suo antigrafo era o lacunosa o del tutto diversa. 
ctissima», ma perché la serie delle riscritture testimonia un'opzione netta in favore dell'endiadi di contro alla terna di attributi promossa a testo da Pistelli; in questo senso, si potrà considerare anche la dittologia «magnifico atque victorioso» che onora Cangrande della Scala nella XIII epistola, sebbene lo statuto illustre ma non imperiale del destinatario renda il riscontro meno probante di quelli destinati alla consorte di Enrico VII.

Dovendo quindi, per le prime due ragioni illustrate sopra, compiere quella selezione che Pistelli non si sentì di operare, e sebbene la situazione stemmatica, assente $V$, non sia di per sé tale da assicurare la selezione, la scelta non mi pare che poter cadere in favore dell'accordo di $P, S$, volg. A e volg. B («gloriosissimo et felicissimo»), già promosso a testo da Toynbee. Oltre che la predilezione dantesca per l'endiadi in questa sede, lo consigliano d) l'inopportunità di promuovere a testo la singularis di un testimone non solo seriore ma che, come il resto dell'epistola VII dimostra, è globalmente meno curato e affidabile, di contro a una lezione di cui è certo il valore storico e compatta la circolazione sia in latino che in volgare; ${ }^{17}$ e) l'anomalia costituita da un attributo ecclesiastico riferito all'imperatore in sede protocollare, e ciò nonostante la fede dantesca nella sacralità dell'Impero; f) il fatto che proprio la fede dantesca nella missione di Enrico VII, quando nota a $M$ o al suo antigrafo, renderebbe il «sanctissimus» facilior per chi copiasse senza avere più familiarità con le norme epistolografiche medievali; g) infine, il fatto che la formula conclusiva della salutatio, che ad alcuni è parsa di sapore papale, e che se così fosse sarebbe impugnabile a conferma della "forzatura" del «sanctissimus», in verità non lo è, come si dirà meglio a breve.

Il resto non presenta problemi ecdotici di rilievo. L'auto-presentazione di Dante quale «Florentinus et exul inmeritus» è anch'essa corrente, benché solo nella micro-tradizione dantesca. Varata nel primo semestre del 1303 nella missiva a Uberto e Guido figli del conte Aghinolfo da Romena, dove l'autore si definisce «exul inmeritus» in corpo testo (II, 3), ${ }^{18}$ e poi codificata dalle salutationes delle epistole III («exulanti Pistoriensi Florentinus exul inmeritus per tempora diuturna salutem et perpetue caritatis ardorem»), V («humilis ytalus Dantes Alagherii florentinus et exul inmeritus orat pacem») e VI («Dantes Alagherii florentinus et exul inmeritus scelestissimis Florentinis intrinsecis», dove il mittente è convenientemente anteposto ai disprezzati destinatari), ai tempi della lettera all'Imperatore questa qualifica suona ormai come una marca distintiva

17 Cfr. la lista di errori, lacune e singulares di ciascun testimone in Mazzoni, Le epistole, pp. 76-77 e in Mazzoni, L'edizione delle Opere latine minori, pp. 159-159 (pp. 100-101 dell'anastatica).

18 Per la datazione, cfr. Indizio, Sul mittente. 
del Dante epistolografo. Lo stesso vale per l'attributo «devotissimus», qui al plurale perché inclusivo dei co-mittenti dell'epistola: su otto occorrenze totali nel corpus dantesco, «devotus» compare sette volte nelle sole epistole e sempre alla forma superlativa. Già nella prima, l'aggettivo qualifica il mittente collettivo nella salutatio («devotissimi filii A. capitaneus Consilium et Universitas partis Alborum de Florentia semetipsos devotissime atque promptissime recommendant»), e si trova ripetuto nel corpo della lettera peraltro in associazione con il tema della pace, oltre che della giustizia (I, 8: «nos filii devotissimi vobis et pacis amatores et iusti»), come avviene nella salutatio della VII. ${ }^{19}$

Lasciando da parte per ora la questione di chi siano i secondi mittenti nominati nella salutatio, meritano attenzione sia l'avverbio sia la relativa con cui sono definiti, e tanto più l'interazione di questi due elementi. Nel lessico dantesco, «universaliter/universalmente» significa 'quanto alla natura propria di qualcosa', come nel vocabolario scolastico coevo, ${ }^{20}$ oppure 'nella sua totalità' (quale antonimo di «membratim»), come appunto in questo luogo. ${ }^{21}$ L'avverbio poi non ha nulla della consistenza tecnica della definizione di «universitas» che identifica i mittenti della prima epistola («Consilium et Universitas partis Alborum de Florentia»), come ordinario nel lessico politico basso-medievale dove il termine vale da sinonimo di «populus», ossia di comunità organizzata e dotata di rappresentanti. ${ }^{22}$ A sua volta, la relativa «qui desiderant pacem» è ambigua, potendosi di per sé intendere come di valore appositivo - il fatto di amare la pace varrebbe allora da qualifica degli «omnes Tusci» - o restrittivo la totalità in questione sarebbe limitata a coloro che amano la pace. I contenuti della lettera - che, tra le altre cose, invita Enrico VII a sradicare la mala pianta fiorentina nemica della missione pacificatrice dell'impero - chiariscono che non può trattarsi che della seconda possibilità. ${ }^{23}$ Vale però rilevare la singolarità di

$19 \mathrm{Si}$ vedano poi il biglietto a nome della Gherardesca di Battifolle dove qualifica la mittente (Ep. IX, 1: «devotissima sua G. de Batifolle Dei et Imperii gratia largiente comitissa in Tuscia palatina») e le tre occorrenze della XIII epistola, in cui manifesta la soggezione e devozione di Dante nei confronti di Cangrande (Ep. XIII, 1: «devotissimus suus Dantes Alagherii florentinus natione non moribus»; 3: «sed ex visu postmodum devotissimus et amicus»; 8: «Liquet igitur quod superius dixi, me scilicet esse devotissimum et amicum, nullatenus esse presumptum»). Per queste osservazioni, cfr. Ferrara, «Devotissimus et amicus», pp. 202-203 (alla nota 19 si dovrà leggere Epist. I).

20 Così in Conv. II, xiv, 9; IV, vi, 8; Mon. I, iii, 2; III, xi, 11.

21 Così in DVE, I, vi, 3; Ep. VII, 1 e 14; Mon. II, vi, 8.

22 Calasso, I glossatori, p. 8.

23 Questa soluzione, per noi piana, non è forse sempre apparsa tale. Se il valore della relativa permane ambiguo nel volgarizzamento A («e universalmente tutti e' toscani che pace desiderano», ed. da Montefusco, Appendice III. I volgarizzamenti, p. 271), il volgarizzamento B pare 
questo sintagma dantesco: in apparenza piano, esso ingenera una tensione tra la presunta totalità a nome della quale il mittente afferma di scrivere e la sua natura de facto parziale perché esclusiva di coloro che, non desiderando la pace, non sono degni di farne parte. Si tratta di una tensione propria a tutta la cosiddetta "politica dell'esclusione" basso-medievale: ${ }^{24}$ il giro sintattico della salutatio dantesca la mette in scena con particolare vigore, così come la enfatizza la dottrina della Monarchia universale quando, dal piano della speculazione teologico-politica, si passi a quello della militanza, come appunto qui avviene.

Infine, riguardo all'ultima formula, ossia al «terre osculum ante pedes», al mandare baci alla terra di fronte ai piedi dell'imperatore, vari commenti recenti informano, sulla scorta del Candelabrum di Bene da Firenze e altre artes dictaminis così come di alcune occorrenze nell'epistolografia medievale, che si tratta di espressione consigliata per rivolgersi al papa; e dunque, dopo il «sanctissimus» già discusso, per la seconda volta Dante trasporrebbe a Enrico VII prerogative retoriche tradizionalmente pontificie. ${ }^{25}$ La formula «pedum oscula beatorum», ossia il bacio dei santi piedi, è in effetti del tutto corrente per il papa, e consigliata in un'opera di riferimento quale la Poetria (o De arte prosaica metrica et rithmica) di Giovanni di Garlandia, familiare al Dante del De vulgari. ${ }^{26}$

risolverlo in una appositiva («e tutti i Toscani universalmente, che pace desiderano», ed. da Montefusco, Appendice III. I volgarizzamenti, p. 275).

24 Riecheggiando il titolo di Milani, L'esclusione dal Comune. Per una riflessione trasversale sulla problematica dell'esclusione si vedano gli studi editi nella prima sezione di Brilli, Fenelli, Wolf, Images and Words in Exile, pp. 3-128, e quelli raccolti in Menzinger, Cittadinanze medievali.

25 Cfr. Ep. (Pastore Stocchi), p. 55: «D. riecheggia piuttosto la salutatio riservata al Pontefice, che i formulari suggerivano appunto di concludere con pedum oscula beatorum, ad pedes paternitatis solotenus prosternendo e sim.» con rinvio alla Summula di Bernoldo di Kaisersheim (Rockinger, Briefsteller, vol. 2, p. 856) che, redatta tra 1312-1314, è pressoché coeva all'epistola. Dello stesso avviso la chiosa in Ep. (Villa), p. 1551 e in Ep. (Baglio) p. 155, che rinvia a titolo di esempio a Bene da Firenze, Candelabrum III, 40, 7; allo Pseudo Brunetto, Sommetta, p. 215; e a una lettera di Enrico VII a Clemente V del 1309 (Const. 4/1, n² 293, p. 255).

26 Per averne la misura, nei volumi delle sole Constitutiones et acta publica dei MGH, $L L$, che includono epistole e documenti dal X al XIV secolo, si contano poco meno di 80 occorrenze di questa formula, tutte riferite al pontefice, le più antiche delle quali rimontano al pontificato di Gregorio IX (2, p. 161; p. 223; p. 313). Parallelamente, all'inizio del XIII secolo, Giovanni di Garlandia consigliava, nel capitolo dedicato appunto alla salutatio, «aliquis prelatus dicit summo Pontifici propter reverenciam loco salutis, "Talis Episcopus licet indignus (vel "humilis minister") tam debitam quam devotam obedientiam”, vel "devotissima pedum oscula beatorum”, vel "Sanctitatis Sue pedibus prostratrum”» (Giovanni di Garlandia, Poetria, V, 275-279, p. 98; o nell'ed. Mari, p. 925). Circa la frequentazione dantesca della Poetria, più che la breve voce di Beggiato, Giovanni di Garlandia, in ED, cfr. i riscontri nel De vulgari eloquentia (Tavoni), a I, xviii, 4; II, iv, 3-5; II, vii, 6; II, x, 2) e Marguin-Hamon, Arts poétiques médiolatins. 
Tuttavia, e al di là del fatto che almeno in un caso duecentesco questa formula si trova eccezionalmente utilizzata per un imperatore, ${ }^{27}$ la dantesca («terre osculum ante pedes») è però diversa e di essa si conoscono varie attestazioni riferite a destinatari sì eminenti ma laici. Già l'edizione Toynbee (p. 30), criticando la lezione «osculantur pedes» promossa a testo da Witte, allegava due occorrenze di poco posteriori a Dante, in epistole inviate rispettivamente dal Comune di Lucca e da quello di Siena a Roberto d'Angiò nel $1312 .{ }^{28}$ Si possono inoltre aggiungere altre occorrenze anteriori di cui due, a mio parere, di un certo interesse. Sono tratte da lettere destinate ad Alberto d'Asburgo, l'Alberto «tedesco» dell'apostrofe del VI del Purgatorio, rispettivamente dal Comune di Arezzo (nel 1301-1302) ${ }^{29}$ e dal podestà di Pistoia, Comune di Arezzo e altri soggetti non identificabili per via di una lacuna (il 17 maggio 1305) ${ }^{30}$ per dichiarare la propria fedeltà in vista di una prossima ma mai realizzata spedizione italiana. Dato il destinatario (un imperatore che si auspicava presto coronato), l'obiettivo della corrispondenza (i preparativi della missione), nonché la provenienza (prossima geograficamente e ideologicamente a quel Casentino dove Dante risiede quando scrive l'epistola VII), questi riscontri, tra i vari disponibili, meritano attenzione quali possibili indici di una tradizione epistolografica locale cui Dante potrebbe aver voluto collegarsi. ${ }^{31}$ Da questo breve excursus si ricava che

27 Così il conte Enrico di Malta, cantato da Peire Vidal, s'indirizzava a Federico II: «suo invictissimo ac precellentissimo domino Friderico illustri Romanorum imperatori semper augusto, Ierusalem et Scicilie regi comes Henricus suus devotus subditus flexis genibus oscula pedum» (Die Aktenstücke zum Frieden von S. Germano 1230, p. 108). Si tratta dell'unico caso che mi sia stato possibile individuare.

28 «Serenissimo Principi dno. Roberto dei gratia Illustri Ierusalem et Sicilie Regi, Ducatus Apulie, etc. (...) populus et Commune Civitatis Lucane, terre obsculum ante pedes» (del 13 Ottobre 1312, ed. in Acta Henrici VII, vol. I, p. 232) e «Serenissimo Principi domino Roberto, dei gratia Ierusalem et Sicilie Regi, Illustr. Capitanei partis Guelforum Civitatis Senarum, terre obsculum ante pedes» (Acta Henrici VII Imperatoris Romanorum, p. 234), entrambe cit. in Ep. (Toynbee), pp. 87-88, che aggiorna Toynbee, A mispunctuation.

29 «Serenissimo ac triumphanti principi domino Alberto Dei gracia Romanorum regi semper augusto Pochaterra potestas, octo gubernatores et defensores, consilium et commune civitatis Arecii imperii Romani fideles devocionis osculum ante pedes» (Henrici VII Constitutiones, p. 88). 30 «Excellentissimo ac trihumphatori principi domino Alberto Dei gratia Romanorum regi semp[er augu]sto Soffredus de Vergellen(sibus) de Pistorio miles potestas, ‘...〉 XXIIIIor, consilium et comune civitatis Aretii imperii Romani [fideles] terre osculum ante pedes et se ipsos humiliter commendatos» (in Henrici VII Constitutiones, p. 170).

31 Preziosi per la precedente tradizione epistolografica locale Stella, I manuali di epistolografia e Bartoli, I conti Guidi, in cui tuttavia non figurano esempi della formula dantesca. La medesima compare invece in altre produzioni epistolografiche tra XIII e XIV secolo indirizzate a sovrani, ossia (in ordine cronologico) nella missiva di Stefano di S. Giorgio al re di Castiglia, Sancio IV, intorno al 1288 («illustri Rege Castelle, Stephanus devotum terre osculum ante pe- 
l'ultima formula della salutatio della VII epistola, pur se non usuale, non è però un'invenzione dantesca e, soprattutto, non implica nessuna volontà di ritrarre Enrico sub specie papae - non più s'intende di quanto non facesse di per sé il rituale del bacio dei piedi attestato per il Lussemburghese sia da Dante sia da Mussato. ${ }^{32}$

\section{Avventure critiche degli «omnes Tusci qui pacem desiderant»}

Rimane da risolvere la questione più importante: chi sono gli «universaliter omnes Tusci qui pacem desiderant» a nome dei quali, oltre che proprio, Dante s'indirizza a Enrico VII? Nei commenti si registra una certa reticenza nei confronti di questo passo, così come oltre laddove Dante ripete di scrivere anche per innominati altri (par. 9: «nam et ego qui scribo tam pro me quam pro alii»). ${ }^{33}$ Ciò con due sole eccezioni. Secondo il commento di Monti già citato costoro sono «tutti i fuoriusciti toscani: tanto quelli che erano con Dante, a nome dei quali e suo egli scrisse questa lettera, quanto gli altri “cacciati per ogni villa” sparsi e dispersi qua e là per l’Italia» (p.180, e cfr. p. 187). A sua volta, Baglio informa che Dante «si fa portavoce di un gruppo, i fuoriusciti e i ghibellini che tra Forlì e le sorgenti dell'Arno riponevano fiducia nell'azione

des», ed. in Una silloge, $\mathrm{n}^{\circ} 60$, p. 60; ringrazio B. Grévin per la segnalazione); in una lettera del 3 maggio 1305 per Bianca d'Aragona («illustrissime principisse domine Bla.nche, Dei gratia regine Aragonum. Guillelmus de Cuperantia, de Vicecomitibus de Pisis, illustrissimi principis domini regis Karoli consiliarius et familiaris, terre obsculum ante pedes», ed. in Salavert Y Roca, Cerdeña, vol. II, p. 159); infine in una del 4 aprile 1325 per il re di Aragona («sacre regie magestati suus humilis et devotus Michael Stephani (...) humili et devota reverentia terre osculum ante pedes», ed. in Const. 6/1, $\mathrm{n}^{\circ}$ 48, p. 31).

32 Cfr. Ep. VII, 9, dove si ricorda il momento «cum pedes tuos manus mee tractarunt et labia mea debitum persolverunt», e Mussato, Historia augustea III, 8, col. 376.

33 Non commentano le edizioni $E p$. (Witte), p. 30; Ep. (Torri), p. 52 (benché nella prefazione, a firma di P. Fraticelli, si dicesse che «in suo nome e a quello pure degli altri esuli ghibellini toscani prese a scrivergli [a Enrico VII] questa lettera. In essa va dicendo, che i suoi fedeli toscani si meravigliano della sua tarda venuta», p. 49); Ep. (Fraticelli), pp. 488-491, e così nella Ep. (Fraticelli²), pp. 412 e ss., in part. pp. 464 e 466-467; Ep. (Toynbee), pp. 87-88 e 90; Ep. (Del Monte), pp. 771-772; Ep. (Pézard), pp. 767-773; Ep. (Brugnoli-Frugoni), p. 562 e p. 564; Ep. (Jacomuzzi), pp. 400-403, e nulla si trova a riguardo nella nota introduttiva alle pp. 349350; Ep. (Lokaj), pp.630-632 e 636; Ep. (Honess), pp. 69-71 e 72-73; Ep. (Pastore Stocchi), pp. 54-55 e 58; Ep. (Villa), che pure, nel presentare l'epistola, parafrasa il passo come «a nome di tutti i Toscani anelanti alla pace» (p. 1550 e 1551-1552). 
del sovrano, come ha già sottolineato il protocollo: universaliter omnes Tusci» (p. 161). A distanza di quasi un secolo, di comune c'è il suggerimento che Dante scrivesse a nome di un gruppo di fuoriusciti, sebbene oscilli l'identità storica del gruppo e non siano chiarite le modalità concrete di questo farsi "portavoce". Entrambe le osservazioni sono probabilmente veicolate da una tradizione diversa di studi, quella storico-biografica, e a questa occorre rivolgersi per meglio comprendere la genesi di tali suggerimenti.

Nell'ambito degli studi biografici la salutatio della VII epistola ha in effetti goduto di una discreta fortuna. Più che alla questione dell'identificazione del gruppo al nome del quale Dante scrive, ci si è interessati a quella del luogo di redazione, indicato da Dante attraverso la perifrasi «in Tuscia sub fonte Sarni». ${ }^{34}$ In Casentino, dunque, ma ciò non dice in quali dei molti castelli del giogo appenninico in mano ai vari pronipoti della «buona Gualdrada» (Inf. XVI, 37) Dante si trovasse precisamente. La scelta è caduta in particolare tra il castello di Poppi, possedimento di Guido da Battifolle, dove certamente Dante si trova pochi mesi dopo quando scrive il terzo dei biglietti a Margherita di Brabante a nome della Gherardesca, ${ }^{35}$ e quello di Porciano, in mano al ramo dei Guidi di Modigliana-Porciano e che, trovandosi una ventina di chilometri più a nord e alle pendici del Falterona, meglio pare convenire alla perifrasi dantesca. Nell'ambito di questa discussione, si è posta quindi anche la questione di chi siano gli «omnes Tusci». Il primo studioso a me noto a esprimersi in merito fu Francesco Torraca. In una lunga recensione al lavoro di Oddone Zenatti su Dante e Firenze, Torraca polemizzava con un'ipotesi di matrice britannica secondo cui Dante si sarebbe trovato in Casentino in veste di ambasciatore di Enrico VII, facendo notare di contro che la salutatio della VII epistola «vuol dire che il Casentino, onde l'epistola fu inviata, accoglieva altri esuli fiorentini. Forse vi si erano radunati pur allora, nella previsione di prossimi avvenimenti, per poter essere più presto e più facilmente informati di ciò, che Firenze meditava e faceva; forse vi dimoravano già prima della discesa di Arrigo». ${ }^{36}$ Assente in Robert

34 Ep. VII, 31, parzialmente parallelo alla dicitura di VI, 27: «in finibus Tuscie sub fontem Sarni».

$35 \mathrm{Ep}$. X, 6: «missum de Castro Poppii xv Kalendas Iunias, faustissimi cursus Henrici Cesaris ad Ytaliam anno primo».

36 Torraca, rec. a Zenatti, p. 153. Il riferimento polemico è costituito da Wicksteed, Gardner, Dante and Giovanni del Virgilio, p. 60 («it is by no means certain what had brought Dante to the Casentino, or in what castel he adressed these letters, from the "confines of Tuscany under the source of Arno" and from "Tuscany under the source of the Arno". It is not improbable that he had been entrusted with a mission from the Emperor himself to the Conti Guidi, or had at least accompanied some such embassy; Henry had been sending legations far and wide to seek aid for the imperial cause, and they were sometimes accompanied by Florentine»). In favore dell'ipotesi Torraca adduceva la notizia della presenza in Casentino di Ubaldino de' 
Davidsohn, ${ }^{37}$ l'idea che Dante scriva a nome di precisi personaggi storici si riaffacciò in un saggio di Ernesto Sestan, che suggerì d'identificare il co-mittente (e forse anche committente) in Guido di Battifolle, ${ }^{38}$ e fu abbracciata da Giorgio Petrocchi. Nella Vita di Dante, esprimendosi a favore di un soggiorno a Poppi, Petrocchi notava che «scrivendo tam pro me quam pro aliis [Dante] mostra di esser portavoce di tutta la comunità degli esuli fiorentini», e che «finché Dante fu a Poppi, l'ambiente politico si mostrava propenso a che un suo illustre ospite si facesse banditore delle finalità politiche della spedizione imperiale». ${ }^{39}$

Da qui la notizia diviene patrimonio comune. Francesco Mazzoni sottolineava le «profonde connessioni con l'ambiente politico dei conti Guidi; naturalmente con quelli, fra i vari rami della casata, che, almeno a quell'altezza cronologica, eran schierati con la parte imperiale», senza tuttavia dettagliare né sbilanciarsi circa un'eventuale committenza della VII epistola, accolta invece da Rosetta Migliorini Fissi sulla scorta di Petrocchi. ${ }^{40}$ Gli stessi argomenti si ritrovano sinteticamente nella Vita curata da Giorgio Inglese, secondo il quale «Dante stavolta parla a nome di un gruppo attivo fra le sorgenti dell'Arno e la vicina Forlì». ${ }^{41}$ Diversamente, essi hanno subito un importante sviluppo nei lavori di Umberto Carpi e, sulla sua scorta, di Marco Santagata. Ne La nobiltà di Dante, esprimendosi a favore della collocazione a Poppi di queste lettere, Carpi affermava non solo che, in questo frangente, Dante si accompagna ad altri fuoriusciti ma che anche svolge funzioni di «intellettuale di corte analoghe a quelle già esercitate per esempio presso Scarpetta Ordelaffi prima e poi presso i Malaspina» presso i conti Guidi. ${ }^{42}$ A sua volta, in Dante. Il romanzo della sua vita, in

Cerchi nel 1310, di cui si discuterà oltre (par. 4). La lettura di Torraca fu forse influenzata da lavori come il grande affresco di Villari, Dante, gli esuli fiorentini dove la tesi relativa all'epistola VII non si trova ma pure si afferma che la Monarchia «divenne allora il programma del partito ghibellino» (p. 168) e che Dante «in questo momento fu come il rappresentante principale del partito imperiale» (p. 175, e cfr. p. 177).

37 Cfr. Davidsohn, Dante, $i$ conti Guidi, cautamente a favore della collocazione a Porciano.

38 Cfr. Sestan, Dante e i conti Guidi, senza pronunciarsi in merito al luogo di composizione, sottolinea la temporanea intesa ideologica tra Dante e i Guidi in questi mesi (p. 351) e suggerisce: «non è affatto da respingere l'ipotesi che la lettera sollecitatoria sia stata a Dante ispirata se non proprio comandata - dal suo ospite il conte Guido di Battifolle, tentennante e timoroso del suo fresco ghibellinismo, se il monarca non venisse presto in Toscana a dargli mano forte contro le pressioni della guelfa Firenze» (pp. 353-354).

39 Petrocchi, Vita di Dante, p. 60a (nell'ed. del 1984 alle pp. 149-150).

40 Mazzoni, Dante e la terra casentinese, p. V (nella rist. anastatica p. 103): a mia conoscenza, Mazzoni non fece mai riferimento a una committenza della VII epistola. Cfr. Migliorini Fissi, Dante e il Casentino, pp. 132-133.

41 Inglese, Vita di Dante, p. 112 (fonte di Baglio, citato supra). Il riferimento a Forlì deriva invece da una notizia trasmessa da Biondo Flavio, della quale si discuterà infra.

42 Carpi, La nobiltà, pp. 511-512, e p. 569. 
un capitolo significativamente intitolato Con i vecchi compagni di lotta, Santagata ha sostenuto che, al tempo della spedizione di Enrico VII, è «più che probabile (...) che Dante sia rientrato, per così dire, nei ranghi e che di nuovo abbia messo a disposizione dei gruppi "bianchi” e ghibellini le sue competenze di dictator e di intellettuale. Potremmo dire che aveva ripreso il suo mestiere di segretario e di addetto alle relazioni esterne», appoggiandosi appunto sulla salutatio della epistola VII. ${ }^{43}$

Rileggendo queste pagine si apprezza, al di là degli addentellati addotti e che si valuteranno oltre, l'evolvere dell'impianto argomentativo. Da un lato, rispetto ai tempi di Torraca e Monti, permane una vaghezza di fondo circa l'identità dei committenti presunti (i Guidi? Quali Guidi? I fuoriusciti? Entrambi?) e di quel che significa che Dante fosse loro "portavoce" (con un mandato ufficiale? Di che tipo?). Dall'altro, la tesi si è progressivamente complicata, cosicché la fisionomia intellettuale e professionale di Dante oscilla senza soluzione di continuità tra il "portaparola" di una rinata Universitas alborum (come ai tempi della I epistola), l'"intellettuale di corte" dei Guidi, e ancora il "segretario" di una qualche cancelleria palatina in Casentino. Per infime che queste differenze possano sembrare sono invece fondamentali in sede storico-biografica e anche, come vorrei mostrare, hanno ricadute consistenti sull'interpretazione e dei testi e dei gesti testuali danteschi. Conviene quindi sforzarsi di valutare se è plausibile ritenere il redattore della VII epistola il rappresentante di una collettività empirica, fosse essa costituita dai fuoriusciti toscani (fiorentini o an-

43 Santagata, Dante, p. 223. La tesi è ribadita oltre: «anche questa epistola non va considerata il gesto isolato di chi, per essere stato ricevuto in udienza, si sente autorizzato a impartire consigli, se non lezioni, a un imperatore; del resto, Dante stesso dice di scrivere a nome suo e di altri. Fa parte di una strategia elaborata proprio in quei giorni dai fuoriusciti toscani per convincere Enrico, attraverso pressioni congiunte, a imprimere un corso diverso alla sua spedizione» (p. 243). Connesso a ciò è l'argomento secondo cui la lettera non potesse essere scritta che a Porciano, presso i Guidi di Modigliana, poiché toni e contenuti non sarebbero stati compatibili con l'orientamento politico di Guido di Battifolle (pp. 240-243). L'argomento è però reversibile: l'invio di queste lettere, che si devono credere pubbliche, non crea infatti problemi a Dante quando, almeno il 18 maggio 1311, trova rifugio a Poppi e scrive all'imperatrice a nome di Gherardesca moglie di Guido da Battifolle (Ep. VIII, IX e X). Santagata discute oltre questo soggiorno ma, in linea con quanto esposto sopra, sminuisce il significato politico delle missive lì composte: «l'accenno alla spedizione di Enrico non poteva mancare, ma, confinato com'è all'interno di un quadretto familiare e ridotto a motivo di soddisfazione privata, è privo di mordente politico. Guido di Battifolle non voleva correre rischi, sapendo che quella lettera sarebbe finita negli archivi della cancelleria imperiale» (Santagata, Dante, p. 245). Per completare il quadro dei maggiori biografi recenti, il riferimento a una committenza esterna è assente nei passi consacrati alla epistola VII da Malato, Dante, pp. 205-206; Pasquini, Vita, pp. 70-71 e Gorni, Dante, p. 224. 
che di altri centri), dai Guidi (uniti o solo alcuni rami della famiglia), o ancora da entrambe queste categorie. Oltre alla salutatio dell'epistola VII (ma è appunto ciò che occorre interpretare), sono stati addotti alcuni argomenti che discuterò nel seguito distinguendo, per comodità, quelli in merito alla mobilitazione politica dei conti Guidi in favore di Enrico VII da quelli circa la presenza di un gruppo di fuoriusciti in Casentino al tempo della redazione della lettera.

\section{Dante "portavoce" dei conti Guidi?}

Consideriamo dunque se è credibile che nell'aprile del 1311, tutto all'opposto di ciò che voleva l'antica leggenda secondo cui Dante sarebbe stato prigioniero dei Guidi, ${ }^{44}$ fosse da loro incaricato di scrivere all'imperatore per sollecitarne la discesa in Toscana. Quale indice della mobilitazione dei conti in favore di Enrico VII è consuetudine riferirsi ai fatti narrati nella relazione indirizzata a Clemente V da Niccolò di Butrinto, ossia Nicolas de Ligny (m. 1316), un frate dell'ordine domenicano, originario di Toul in Lorena e al servizio di Enrico VII al tempo di fatti, sebbene prossimo anche alla curia pontificia. ${ }^{45}$ Riassumendo per sommi capi, Niccolò racconta che lui e Pandolfo Savelli furono inviati dall'imperatore in Tuscia nel settembre del 1311. La spedizione diplomatica, la seconda rivolta alla Tuscia dopo quella del 1310, aveva tra i suoi obiettivi la città di Firenze, di cui pure ben si conosceva la posizione anti-arrighiana. Per limitarsi a un solo esempio, l'estate precedente erano state intercettate alcune

44 A questa leggenda locale, secondo cui Dante sarebbe stato rinchiuso nel castello di Porciano, si riferiva Troya, Del Veltro allegorico, pp. 123-124 che appunto rinveniva nell'epistola VII una possibile ragione di questa ritorsione.

45 Niccolò di Butrinto, Relatio, pp. 519 e 522 e ss. Un ricordo dello stesso episodio che qui interessa si legge anche in Villani, Nuova cronica, X, 26 e, sulla sua scorta, in Marchionne di Coppo Stefani, Cronaca fiorentina, rubr. 283, pp. 106-107; ne tace invece Compagni, Cronica, che ricorda però l'ambasceria precedente di Luigi di Savoia del luglio del 1310 (p. 139) ma collocandola dopo l'arrivo dell'imperatore a Pisa nel 1311, segno forse in una qualche confusione tra i due episodi. Di quest'episodio si veda anche la parafrasi, pur a tratti libera, di Davidsohn, Storia di Firenze, III, pp. 605-614. Ne discorre invece solo rapidamente Bowsky, Henry VII, p. 140, che pure attinge abbondantemente a Niccolò come fonte per altri dettagli (e del resto, di tutti i Guidi, Bowsky menziona solo una volta en passant Ildebrandino di Romena, p. 154). Per una valutazione della relatio come fonte storica cfr. Franke, Kaiser Heinrich VII., pp. 159-201; Zanella, L’imperatore tiranno, pp. 43-56 e i contributi raccolti in Varanini, Enrico VII e il governo. Nella dantistica, dopo gli accenni di Davidsohn, Dante, i conti Guidi e Sestan, Dante e i conti Guidi, la relatio è stata messa in valore da Carpi, La nobilità, vol. II, pp. 664666, seguito da Santagata, Dante. 
missive spedite dal comune di Brescia alla città toscana contenenti richieste di supporto alla resistenza anti-imperiale. ${ }^{46}$ Partiti tra il 15 e il 17 ottobre 1311, i due legati si avviano per la via Emilia ma, giunti in prossimità di Bologna, sono informati del rifiuto del Comune di farli passare e costretti a cercare riparo sugli Appennini, in un castello del vescovo nei pressi di Bologna sulla via per Firenze. ${ }^{47}$ L'indomani ripartono e dopo varie vicissitudini, ormai giunti alla Lastra (25 ottobre 1311, o più precisamente Montughi, secondo Villani), inviano un messo a Firenze che al ritorno li informa che anche i fiorentini hanno emesso un provvedimento di bando ai loro danni. ${ }^{48}$

Tuttavia, dei membri della famiglia degli Spini, in particolare Avvocato Spini, intrattenendo relazioni di lunga data con la Curia pontificia e in particolare con il pontefice Onorio IV Savelli (1210-1287), zio di Pandolfo, sono preoccupati per la sorte degli ambasciatori: durante la notte inviano loro delle lettere per avvisarli del pericolo incombente e l'indomani convincono il Comune a inviare tre delegati (un armato rappresentante del potestà, un popolare del capitano e Avvocato Spini) per proteggerli dai fiorentini, che hanno già dato l'assalto al palazzo in cui i legati sono ospitati. ${ }^{49}$ Dopo una lunga consultazione si decide che, pericolose e inagibili tanto Firenze quanto Bologna, l'unica soluzione è passare «per quamdam viam que ibat ad terras comitum Guidonum, qui habitant inter Bononiam, Romandiolam et Aretium». ${ }^{50}$ Così è fatto. Il 27 ottobre 1311 Niccolò e Pandolfo sono lasciati dai loro protettori «in quodam oppido in quo Florentini et comes Tegrinus habebant dominium», ${ }^{51}$ e quest'ultimo, il conte

46 Niccolò di Butrinto, Relatio, pp. 511-512.

47 Forse Sasso Marconi, stando alla descrizione: «in strata intellectis hiis que acta sunt circa eum, dimissa Bononia a sinistris, per castrum Episcopi posuimus nos in Alpibus per viam horribilem, et de nocte multum tarde applicuimus ad unum castrum inter Florentiam et Bononiam in via recta, quod castrum a Bononia distabat per septem miliaria», ossia poco meno di 11 chilometri (Niccolò di Butrinto, Relatio, p. 522).

48 Cfr. Niccolò di Butrinto, Relatio, p. 523.

49 Cfr. Niccolò di Butrinto, Relatio, pp. 523-524. Malgrado la protezione offerta agli ambasciatori in questa occasione, Avvocato Spini figurerà tra i ribelli condannati da Enrico VII il 23 febbraio 1313 (Henrici VII Constitutiones, pp. 933-951, a p. 945; cfr. Davidsohn, Storia di Firenze, III, p. 610, nota 2). Non ne trovo menzione in Tripodi, Gli Spini, che si concentra sulle generazioni posteriori.

50 Niccolò di Butrinto, Relatio, p. 525.

51 Niccolò di Butrinto, Relatio, p. 525. Secondo Davidsohn, Dante, i conti Guidi, p. 223, si tratta di Ampinana, a una cinquantina di chilometri. Questo castello fu comprato a patti da Firenze per 3.000 fiorini nel 1291 e distrutto (cfr. Villani, Nuova Cronica, VIII, 150); ai Guidi rimanevano però «i diritti signorili sugli uomini di Ampinana e la signoria sui villaggi vicini» (Bicchierai, Guidi, Manfredi): ciò corrisponde bene alla descrizione di Niccolò come di un luogo di frontiera e dalla giurisdizione mista tra la città e i Guidi. 
Tegrimo II di Modigliana-Porciano (1240/1250-1315), uno degli undici figli di Guido di Modigliana e di Adelasia di Bonifazio di Panico, li recupera appunto qui per condurli a San Godenzo una volta scesa la notte. ${ }^{52} \mathrm{Da}$ qui, la comitiva procede «ad alium locum», ${ }^{53}$ dove è raggiunta da due fratelli del conte Tegrimo, nominatim Tancredi (m. 1318 ca.) e Bandino (m. 1313) più, in un secondo tempo, anche da un quarto fratello, Ruggeri (m. 1318). A questo punto, in una sorte di descrizione generale della famiglia, Niccolò menziona anche gli esponenti degli altri rami dei Guidi, e in particolare il ramo da Romena, il conte Guido Salvatico dei Guidi di Dovadola (m. 1316 ca) e Guido di Battifolle di Poppi (m. 1322/1323). Ricorda poi i giuramenti di fedeltà all'imperatore e le promesse di raggiungerlo quanto prima (o almeno non appena fosse giunto in Tuscia; Enrico VII è in questo momento a Genova) e di sostenerlo secondo le diverse disponibilità, che tuttavia solo pochi mantennero.

Isti omnes [scilicet Tegrimo, Tancredi, Bandino e Ruggero, ossia i Guidi di ModiglianaPorciano] dicuntur de comitibus Guidonibus, et sunt comites palatini; et eiusdem domus sunt comes de Batefole et comes Salvaticus. Item comites de Romena omnes vocant se comites palatinos, et magnos redditus habent in Romandiola. Inter istos quidam sunt Guelphi, et illi sunt ditiores, videlicet comes Salvaticus et comes de Batifole et comes Bandinus. Omnes iuraverunt nobis fidelitatem et venire ad dominum suum personaliter, vel mittere, si impediti essent, statim quod esset in Tuscia, et ipsum associare ad coronam in illa decentia in qua possent secundum suas facultates. Isti omnes nos multum honoraverunt et gaudenter receperunt, et plus Guelphi quam Guebelini, meo iudicio. Tamen non obstantibus fidelitatibus nullus de istis Guelphis venit ad eum in Tusciam vel misit ad renovandum iuramentum, sed dilationem petiverunt et habuerunt usque ad terminum petitum; et in termino petito, iam imperatore existente ante Florentiam, confederati sunt publice cum Florentinis, cum ipsis in Florentia existentes. Qui erant Guebelini inter istos, vel venerunt qui potuerunt vel miserunt, et cum eo fuerunt usque ad mortem suam. ${ }^{54}$

L'interpretazione complessiva è in verità delicata e dipende da se s'intenda l'«omnes iuraverunt nobis fidelitatem etc.» come riferito ai soli personaggi che

52 Cfr. Bicchierai, Guidi, Tegrimo. San Godenzo dista circa 15 chilometri da Ampinana, tragitto percorribile in qualche ora anche se, come è qui il caso, la compagnia include servi a piedi (cfr. Niccolò di Butrinto, Relatio, p. 525: «et in nocte dictus comes Tigrinus duxit nos inter Alpes ad unum burgum suum qui vocatur burgus Sancti Gaudentii, et mutuavit nobis equos quot potuit pro familia, que erat pedes»). Lì forse i legati furono ospitati nel Palazzo dello Specchio dei Guidi di Modigliana, «così detto per il raro - per allora - uso di vetri alle finestre che così brillavano al sole» (Bicchierai, Guidi, Guido, e come già suggeriva Davidsohn, Dante, i conti Guidi, p. 223).

53 Niccolò di Butrinto, Relatio, p. 525. Difficile identificare questo luogo: nel percorso lungo l'Arno verso il Valdarno inferiore, dove proseguirà la spedizione di Niccolò, si sarà potuto trattare di Porciano, Romena, Poppi, e neanche si possono escludere Stia e Pratovecchio dato che Niccolò parla di un «locum» e non di un castello.

54 Niccolò di Butrinto, Relatio, pp. 525-526. 
Niccolò ha esplicitamente detto presenti alla riunione descritta sopra (i conti di Modigliana-Porciano), oppure a tutti i Guidi citati nella descrizione della famiglia (ossia anche i rami di Battifolle, Dovadola e Romena). Da ciò deriva l'oscillazione presso gli storici tra chi ritiene che anche Guido di Battifolle e Guido Salvatico fossero presenti e giurassero fedeltà a Enrico VII e chi si mostra più cauto. ${ }^{55}$ Anche è possibile che Niccolò si riferisca qui cumulativamente a più incontri avvenuti in Casentino dal 27 al 31 ottobre, ma vale notare che non menziona mai Guido Novello II (o il Giovane, o di Raggiolo), di cui pure sono noti il ghibellinismo e la militanza pro Enrico VII. ${ }^{56}$ Come che fosse, dal Casentino la spedizione prosegue poi verso il territorio del vescovo di Arezzo, un altro membro della famiglia dei Guidi del ramo di Romena, Ildebrandino (1257/12651312). Il vescovo li accoglie prima a Civitella in Val di Chiana (31 ottobre 1311), che diviene la base logistica dove gli ambasciatori convocano i rappresentanti di vari comuni della Tuscia sud-orientale, ${ }^{57}$ e poi ad Arezzo, dove citano nobili guelfi e ghibellini di Toscana. ${ }^{58}$ Senza bisogno di seguire gli ambasciatori, che con varie difficoltà approdano nel grossetano e poi, via mare, ritrovano la corte di Enrico VII ormai di stanza a Pisa (cfr. figura 1), torniamo all'episodio casentinese.

Come si è detto, questi tre giorni sono stati ritenuti significativi non solo di una temporanea convergenza dei Guidi in sostegno di Enrico VII, ma anche del fatto che presso di loro e per loro Dante avrebbe scritto al lussemburghese nell'aprile del 1311. Ora, si tratta senza dubbio di una testimonianza rivelatrice ma di un'atmosfera, mi pare, diversa da questa. Occorre infatti tenere presente non solo che i fatti narrati sono di sei mesi posteriori alla redazione dell'epistola di Dante, ma anche di due altri dati che emergono chiaramente quando si esamini l'episodio nel complesso della relazione di Niccolò. Il primo: i Guidi di Modigliana-Porciano entrano in scena solo grazie alla mediazione degli Spini

55 In favore del primo partito cfr. Davidsohn, Storia di Firenze, III, p. 612; Sestan, Dante e $i$ conti Guidi, p. 350; del secondo Bicchierai, Guidi, Guido Salvatico e Bicchierai, Guidi, Guido.

56 Lo dice probabilmente presente Bicchierai, Guidi, Guido Novello (il Giovane), né è impossibile che, tra tanti conti, il legato francese dimenticasse di menzionarne qualcuno.

57 Tra cui Arezzo, Cortona, Borgo S. Sepolcro (i cui legati si presentano ma non prestano giuramento), Montepulciano, Lucignano, San Savino, Chiusi, Città della Pieve (che però non risponde) e Castiglione Fiorentino (cfr. Niccolò di Butrinto, Relatio, p. 526).

58 Sono menzionati gli Alberti di Mangona, Uguccione Della Faggiuola, i Pazzi di Valdarno, gli Uberti (ma da correggere in «Ubertini», come suggerito da Davidsohn, Storia di Firenze, III, p. 613, nota 3), i Tarlati di Pietramala e, a conferma dell'orientamento sud-orientale strategico in vista della pianificazione della discesa a Roma, «generaliter omnes et quemlibet per se qui erant nobiles in districtu Florentino, Senensi, Aretino et Clusino» (cfr. Niccolò di Butrinto, Relatio, p. 527). 


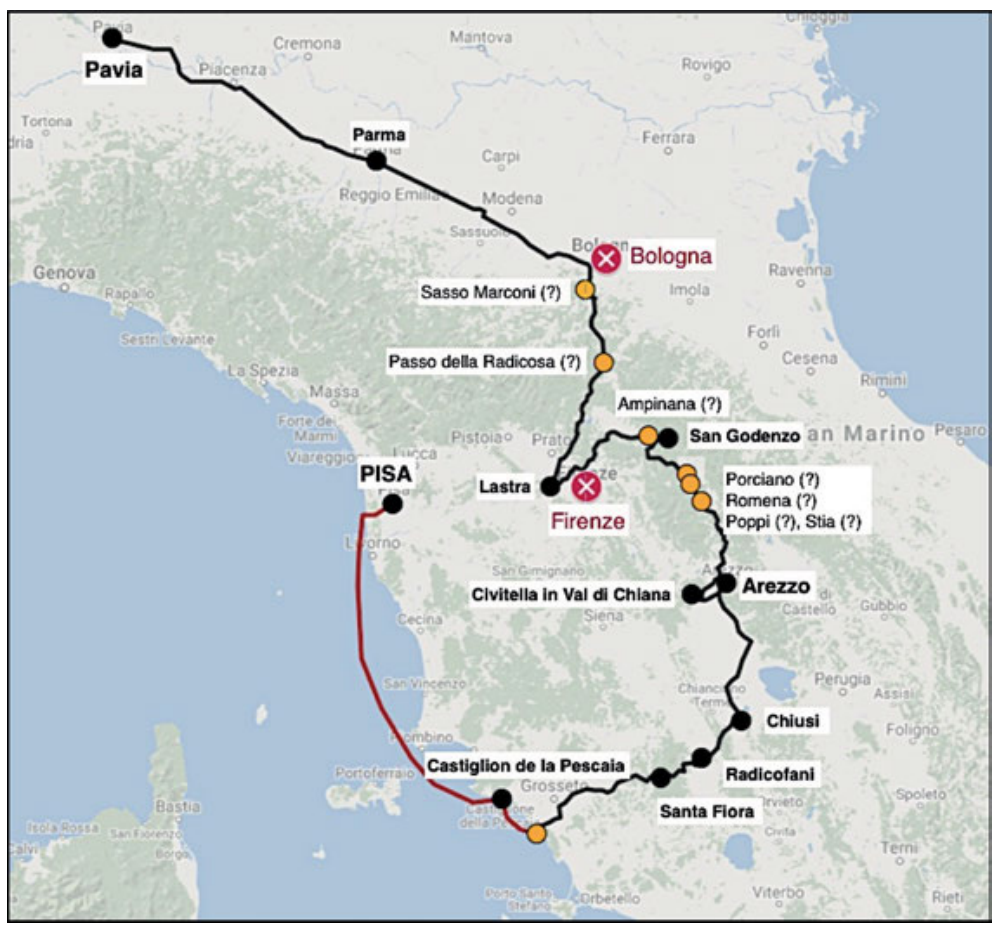

Figura 1: Itinerario di Niccolò di Butrinto e Pandolfo Savelli (10/1311).

di Firenze, che sono famiglia nera e interessata alla sorte del Savelli per motivi più affaristici e relazionali che ideologici. ${ }^{59}$ Il secondo: l'episodio casentinese costituisce per i legati una vera e propria deviazione; una deviazione imposta dai bandi di Bologna e Firenze e decisa non da loro, che avevano invece chiesto un lasciapassare per Firenze, bensì dai tre fiorentini mandati dal Comune e seduta stante. ${ }^{60}$ Nell'ottobre del 1311, insomma, i Guidi di Modigliana-Porciano

59 Tanto più persuasivo suona il suggerimento di Davidsohn, Storia di Firenze, III, p. 605 che Enrico avesse scelto i legati tenendo conto della prossimità alla Curia dell'uno (Niccolò) e della famiglia dell'altro (Pandolfo).

60 Il racconto di Niccolò su questo punto, essenziale per cogliere la dinamica degli eventi, è chiarissimo: «isti tres [scilicet i tre inviati del Comune di Firenze] venerunt ad nos; et partem de equis, partem de somariis in via invenientes, quia ad civitatem ducebantur, violenter de manibus eorum [scilicet dei fiorentini che avevano dato l'assalto al palazzo dove si trovavano i legati] acceperunt, et nobis ipsos restituerunt cum hiis verbis, quod quantum vitam nostram diligebamus, quod in continenti recederemus, et quod ipsi laborarent quod omnia rehaberemus. Nos voluimus eis exponere ambassiatam nostram, noluerunt audire, litteras vestras ostendere, noluerunt videre. Quid plura? Rogavimus eos quod nos permitterent de nocte tran- 
intervengono non spontaneamente ma su richiesta di una famiglia fiorentina intesa a salvaguardare i propri interessi, ossia a sbarazzarsi dei legati imperiali rapidamente e con il minimo danno; per di più, essi non costituiscono gli interlocutori privilegiati degli ambasciatori di Enrico, che difatti non avevano previsto di passare dal Casentino né ci pensano una volta trovatisi nel cul de sac della Lastra. In questo frangente, relativamente eccezionale, i conti accolgono i legati e prestano giuramento: si tratta dell'incipit di una militanza che solo Tancredi, tra i sicuramente presenti, confermerà e, più in generale, la dinamica dell'episodio fa escludere che questo ramo della famiglia avesse giurato fedeltà a Enrico in precedenza - altrimenti si sarebbe parlato di un rinnovare il giuramento - o che si possa retrodatare la sua mobilitazione a prima di questa data. ${ }^{61}$

Tornando a Dante, se nell'ottobre del 1311 così stavano le cose dal lato dei Guidi di Modigliana-Porciano, in che modo ciò può dimostrare che, sei mesi prima, egli svolgesse funzioni di "portavoce”, "intellettuale di corte” o "segreta-

sire per Florentiam, et nos bene custodirent ne aliquid loqueremur. Negaverunt semper, dicentes quod in mandatis habebant ut nos reverteremur unde veniebamus. Ille antiqu[u]s Advocatus de Spinis dixerat nobis ad partem quod nullo modo induceremur reverti per Bononiam vel suum districtum, quia iam erat eis mandatum quod nos expulsi de Florentinorum districtu eramus, et quod de nobis facerent sicut de publicis hostibus, ut de cetero nullus auderet intrare. Nos vilitatem, miseriam et fatuitatem Bononiensium cognoscentes diximus quod, si nos deberent interficere, nunquam per Bononiam reverteremur. Deliberatione habita inter eos [scilicet i fiorentini] magna, posuerunt nos per quamdam viam que ibat ad terras comitum Guidonum» (Niccolò di Butrinto, Relatio, pp. 524-525).

61 L'unico sostenitore di Enrico VII fra i Guidi di Modigliana-Porciano fu Tancredi, che seguì l'imperatore a Roma, quindi a Firenze, e figura come testimone in vari atti d'importanza del 1313 (cfr. Const. 4/2, n 914, pp. 927-929; n 915, pp. 930-932; n 916, pp. 932-951; n 946, pp. 985-990). Lo stesso anno, il 31 marzo, Enrico VII lo ripaga per i suoi servizi donandogli i beni del fratello Bandino, morto senza eredi legittimi, per il fatto che costui «[cum] de bonis et iuribus, quae in Tuscia dudum ab Imperio tenuit a nobis homagium facere, ac fidelitatem praestare infra, vel post tempus a iure statum, et ad exercitum nostrum supra Florentiam prout habuit in mandatis a nobis, accedere, vel mittere contumaciter praetermiserit» (il privilegio del 1313 è conservato in uno del 1355 a favore dei Guidi, ed. in Delizie, vol. VIII, pp. 110-113, cit. a p. 111; cfr. Davidsohn, Dante e i conti, p. 223-224; Bicchierai, Guidi, Tegrimo). Non più caldi si mostrano Tegrimo e Ruggeri che, all'assedio di Firenze, si schierano con la città e anche osteggiano la successione a Tancredi dei beni di Bandino (Bicchierai, Guidi, Tegrimo). La disputa sorta a questo proposito è ricordata nel testamento di Tegrimo del 1315 (citato da Pirillo, La signoria, utile per apprezzare la lunga durata dei conflitti interni a questo ramo della famiglia, e discusso da La Roncière, Diversi conti Guidi), così come in quello della contessa Bice di Guido di Battifolle (cit. ivi, p. 457, nota 25). Pare così da rettificare Santagata, Dante, p. 248 (secondo cui tutti i Guidi di Modigliana-Porciano sarebbero stati ghibellini tranne Ruggeri) ma anche Sestan, Dante e i conti Guidi, p. 350 (secondo cui l'accoglienza nell'autunno del 1311 presuppone che i Guidi di Modigliana-Porciano avessero giurato fedeltà già nell'autunno del 1310, o poco dopo). 
rio" presso di loro e che componesse l'epistola per il Lussemburghese dietro loro richiesta? Il ragionamento poi vale a fortiori per gli altri rami dei conti Guidi, fossero o meno fisicamente presenti all'incontro narrato da Niccolò: sia quelli in seguito favorevoli a Enrico, come Aghinolfo II di Romena e Guido Novello di Reggiolo, ma che non risultano mobilitati per la causa imperiale prima dell'autunno del 1311, ${ }^{62}$ sia, e tanto più, quelli che, come Guido da Battifolle e l'anziano Guido Salvatico, a quest'epoca temporeggiano in attesa di conoscere le evoluzioni dello scacchiere e che, al momento venuto, si schiereranno in favore di Firenze contro l'imperatore. ${ }^{63}$ La terra dei Guidi nell'aprile del 1311 non presenta, in nessuno dei suoi castelli, segni né manifesti né tanto meno compatti di una mobilitazione filo-arrighiana ed è più probabile che vi vigesse un'attesa fluida, tra speranza e sospetto nei confronti della spedizione imperiale, e sospesa tra il dubbio se il lussemburghese sarebbe mai arrivato fin lì e la valutazione del partito migliore da prendere, nell'intrico delle convenienze e opportunità che legano e oppongono i Guidi tra loro e con i centri limitrofi.

A queste considerazioni storiche conviene poi aggiungerne un'altra di carattere retorico e che a me pare, di per sé, definitiva. Se Dante avesse voluto scrivere a nome dei conti Guidi su loro richiesta, si trattasse di un ramo specifico della famiglia o di molti, sarebbe stato del tutto inappropriato - da un punto di vista retorico - nominare dei conti palatini solo dopo sé stesso e appellarli genericamente «omnes Tusci qui desiderant pacem». Come insegnava ad esempio la Poetria di Giovanni di Garlandia:

Si plures mittant simul, digniores preponendi sunt, et si eis mittatur in executione, secundum ordinem loquantur hoc modo: «Ego Magister Iohannes Dilectioni Vestre mitto libellum. Ego W. Subdyaconus Vestre significo Prudencie quod denarios omnes quos postulastis, mittere non possum donec tempus preterierit autumpnale». Sic dicant alii si plures fuerint; si pluribus scribatur, secundum priorem ordinem sua negocia proponantur. ${ }^{64}$

62 Cfr. Bicchierai, Guidi, Aghinolfo e Bicchierai, Guidi, Guido Novello (il Giovane o di Reggiolo). L'unico indizio relativo a un precedente contatto con l'imperatore risale al 12 luglio del 1310 quando, convocato dai legati di Enrico VII (all'epoca a Losanna), ossia Filippo di Rathsamhausen, vescovo di Eichstätt, e Bassiano de' Gaschio o de' Guacii, Aghinolfo invia il notaio Andrea di Betto da Poppi per scusarsi della sua assenza, determinata dalla guerra in corso tra Firenze e Arezzo, incaricandolo di ascoltare le volontà dell'imperatore e «generaliter ad omnia et singula facienda, gerenda et exercenda, que circa predicta et quodlibet predictorum fuerint oportuna» (in Henrici VII Constitutiones, pp. 307-308; cfr. Davidsohn, Storia di Firenze, III, p. 524). Talvolta descritto come un "giuramento di fedeltà" e segno della precoce mobilitazione di Aghinolfo, questo documento sembra piuttosto un'excusatio (come lo intitolava appunto Schwalm) e una dichiarazione di disponibilità, normale quando un conte palatino si trovi così convocato. Ringrazio G. Milani per la consulenza a riguardo.

63 Cfr. Bicchierai, Guidi, Guido e Bicchierai, Guidi, Guido Salvatico.

64 Giovanni di Garlandia, Poetria, V, 289-296, p. 98 (nell'ed. Mari, p. 925). 
L'ispirazione gerarchica della precettistica a riguardo è tanto chiara da far escludere questa possibilità - a meno, ovviamente, che non s'immagini un Dante "segretario" dei Guidi che contravvenga però ad ogni consuetudine retorica, oppure una sorta di cripto-committenza, formulata in modo tale da dissimulare i soggetti da cui emana, donde però il venir meno della stessa motivazione politica del pubblico appello all'imperatore.

\section{Dante "portavoce" dei fuoriusciti in Casentino?}

Accantonata l'eventualità di una committenza dall'alto, è possibile che la salutatio della VII epistola testimoni di una committenza dal basso, da parte cioè dei fuoriusciti che, confluiti in Casentino, seguissero impazienti la missione dell'imperatore? Gli addentellati principali allegati in favore di quest'ipotesi sono tre. Innanzitutto, mentre nel medaglione biografico della Nuova cronica Villani non menziona nessuna committenza per la lettera a Enrico VII e, pur se bene informato sui fatti, nessun manipolo di fuoriusciti in Casentino, ${ }^{65}$ nel Trattatello di Boccaccio l'epistola VII è presentata come il frutto di un'iniziativa concordata con altri oppositori di Firenze. ${ }^{66}$ Il secondo addentellato è offerto dalla notizia, trasmessa da Biondo Flavio, in merito a una lettera che Dante avrebbe indirizzato a Cangrande della Scala a chiosa della risposta data dai

65 Villani, Nuova Cronica, X,136. Per una discussione di questo luogo mi permetto di rinviare a Brilli, Profeti, veri e falsi.

66 Boccaccio, Trattatello (I ${ }^{\mathrm{a}}$ red.), VII, 77-78, pp. 58-59: «per che ripassate l'Alpi, con molti nemici di Fiorentini e di lor parte congiuntosi, e con ambascerie e con lettere s'ingegnarono di tirare lo 'mperadore da l'assedio di Brescia, acciò che a Fiorenza il ponesse, sí come a principale membro de' suoi nemici; mostrandogli che, superata quella, niuna fatica gli restava, o piccola, ad avere libera e espedita la possessione e il dominio di tutta Italia. E come che a lui e agli altri a ciò tenenti venisse fatto il trarloci, non ebbe perciò la sua venuta il fine da loro avvisato: le resistenze furono grandissime, e assai maggiori che da loro avvisate non erano; per che, senza avere niuna notevole cosa operata, lo 'mperadore, partitosi quasi disperato, verso Roma drizzò il suo cammino». La medesima informazione si mantiene nella seconda redazione: «per che, lasciati gli studii e in Italia tornatosi, e con certi rubelli de' Fiorentini congiuntosi, con loro insieme con prieghi, con lettere e con ambasciate s'ingegnò di rimuovere il detto Arrigo dallo assedio di Brescia e di conducerlo intorno alla sua città, estimando quella contro a lui non potersi tenere. Ma la riuscita contraria gli fece palese il suo avviso essere stato vano. Assediò Arrigo la città di Fiorenza; e ultimamente, vana vedendo la stanza, se ne partí e, non dopo molto tempo passando di questa vita, ogni speranza ruppe del nostro poeta, il quale in Romagna se ne passò, dove l'ultimo suo dí, il quale alle sue fatiche doveva por fine, l'aspettava» (Boccaccio, Trattatello (II ${ }^{\mathrm{a}}$ red.), VII, 57-58, p. 131). L'erronea identificazione della città assediata con Brescia tradisce forse un'interferenza villaniana. 
fiorentini agli ambasciatori di Enrico VII nel luglio del 1310 e che, nota a Pellegrino Calvi oltre che a Benvenuto da Imola, sarebbe stata «scritta a nome proprio e degli esiliati di parte bianca». ${ }^{67}$ Infine, il terzo argomento è offerto dalla presenza in Casentino nel luglio del 1310 di un esponente del fuoriuscitismo bianco della prima leva, Ubaldino di Bindo dei Cerchi. ${ }^{68}$

I primi due addentellati presentano le solite incognite delle testimonianze indirette aggravate, nel caso di Boccaccio, dal fatto che il riferimento ai «nemici» (I red.) o «rubelli» (II red.) di Firenze può facilmente spiegarsi come una glossa promossa appunto dalla enigmatica salutatio della VII epistola. Quanto alla testimonianza di Biondo Flavio - generalmente rifiutata per i problemi che comporta nella cronologia dantesca l'ipotesi di un soggiorno a Forlì in data così bassa -, anch'essa si può spiegare rispetto al dato di nostro interesse, ossia l'identificazione dei co-mittenti con una rinata parte bianca, per l'interferenza

67 «Dantes Aldegerius, Forolivii tunc agens, in epistola ad Canem Grandem Scaligerum veronensem, partis Albae extorrum et suo nomine data, quam Peregrinus Calvus scriptam reliquit, talia dicit de responsione supradictae expositioni a Florentinis urbem tenentibus tunc facta, per quae temeritatis et petulantiae ac caecitatis sedentes ad clavum notat, adeo ut Benevenutus Imolensis, quem Peregrini scripta legisse crediderim, Dantem asserat hinc cepisse Florentinos epitheto caecos» (Biondo Flavio, Historiarum decades, II, ix, 342, ed. in appendice a Ep. [Pastore Stocchi], pp. 140-141, così come a Ep. [Baglio], pp. 252-253, da cui è tratta la traduzione in testo). Della conoscenza di questa lettera non resta traccia nel Comentum di Benvenuto da Imola: ringrazio L. Fiorentini per le verifiche a riguardo.

68 Il documento già citato (supra nota 62) è sottoscritto: «in Castro Biblene in camera palatii venerabilis patris domini Ildebrandini Dei gratia episcopi Aretini, in presentia ipsius domini episcopi Aretini, presentibus nobili viro Ubaldino domini Bindi de Cerchis de Florentia, ser Ugolino notario condam Ranuccii de Forlivo et magistro Luca fisico de Biblena, testibus ad hec habitis et vocatis» (Const. 4/1, $\mathrm{n}^{\circ} 360$, p. 308). Secondo lo Pseudo-Brunetto, Ubaldino fu condannato a pena pecuniaria e confino dopo la zuffa in casa Frescobaldi del 1296 (Testi fiorentini, pp. 147-148; cfr. Zorzi, La faida, pp. 104-105; Brilli, Firenze, 1300-1301, p. 122). All'inasprirsi del conflitto, con il cugino Vieri, patrocina Ricoverino, ferito nello scontro di Calendimaggio 1300 (Statuti della Repubblica fiorentina, vol. II, Statuto del Podestà dell'anno 1325, pp. 390-391; cfr. Davidsohn, Storia di Firenze, III, p. 150). Figura quindi tra i condannati da Cante de' Gabrielli il 2 giugno 1302 (Campanelli, Le sentenze, p. 268) e da Gherardino da Gambara il 28 luglio 1302, per aver intavolato trattative con gli Ubaldini e occupato il castello di Gangareta (o Ganghereta) in Valdarno (Campanelli, Le sentenze, p. 295; l'occupazione è confermata dalle Storie Pistoresi, 20, p. 34 e da Paolino Pieri, Croniche, par. 163, p. 79), mentre altri documenti certificano il supporto ricevuto in questi anni da Benedetto XI (Davidsohn, Storia di Firenze, vol. IV, p. 358). Pare verosimile che si tratti dello stesso «Ubaldinus Circli florentinus» che Girolamo Rossi dice marito (intorno al 1317) della contessa Rengarda, figlia di Cavalcaconte di Bagnacavallo (già sposata in prime nozze di Alberto Canali) e padre di un Tano Cerchi (cfr. Hieronymi Rubei Historiae, VI, pp. 532 e 534; nella trad. italiana Girolamo Rossi, Storie, pp. 544 e 546). 
di altri luoghi danteschi, innanzitutto la prima epistola. ${ }^{69}$ Certamente affidabile è invece il documento relativo alla presenza di Ubaldino dei Cerchi a Bibbiena presso Aghinolfo II da Romena nel luglio del 1310, ed è probabilmente opportuno scorgere in ciò il segno della mobilitazione degli esuli del 1302. Si pensi, per fare un solo esempio, che nello stesso torno di mesi si trova a Pisa Giovanni dei Cerchi (m. 1314 ca.) che, mobilitato in prima linea in favore di Enrico VII, sovrintende per conto della città all'allestimento dei doni destinati ai legati imperiali. $^{70}$ Tuttavia, il fatto che nei mesi precedenti la discesa di Enrico si assista a una comprensibile mobilitazione dei fuoriusciti e che uno di essi risulti in Casentino, forse anche in cerca di sostegno in vista degli eventi a venire, non equivale a che il territorio dei conti Guidi fosse il quartier generale dei sostenitori dell'impero. Soprattutto, il fatto che Dante fosse in contatto con altri fuoriusciti, come certamente era, è altro dallo svolgere funzioni di rappresentante di una rinata pars Alborum e dal redigere una lettera emanata da una strategia comune. Si tratta di sfumature importanti in sede di analisi storica e capitali per l'interpretazione dei testi che in questa congiuntura sono concepiti e diffusi.

Alla ricostruzione proposta da chi vede in Dante il volto pubblico dei fuoriusciti osta soprattutto, e nuovamente, la formula della salutatio. Se così fosse, si attenderebbe una menzione esplicita e tecnica della compagine, valga il raffronto con la prima epistola e la sua puntuale definizione di «Consilium et Universitas partis Alborum», opportunamente anteposta a quella del portavoce. La dicitura «omnes Tusci qui desiderant pacem» è poi disallineata rispetto alle modalità politiche e retoriche del conflitto nella penisola all'inizio del Trecento anche per un altro motivo. Le compagnie di esuli si definiscono, infatti, sempre in relazione alla o alle città di provenienza. Lo prova Dante nelle sue autopresentazioni, sempre «Florentinus» per quanto solo di nascita e in esilio, così

69 Sospecciosi circa l'attendibilità della testimonianza Barbi, Sulla dimora, p. 194; Petrocchi, Vita di Dante, pp.150-151; Pastore Stocchi, Epistole, così come in Ep. (Pastore Stocchi), pp. 140-141. Ne ha recentemente difeso l'affidabilità Indizio, Dante secondo i suoi antichi interpreti, pp. 160-161 e pp. 168-171, che su questa base retrodata il soggiorno di Dante a Forlì al secondo semestre del 1310 (e non nel corso del 1311). Inglese, Vita di Dante, pp. 110-111 esclude la possibilità che una tale presa di posizione avvenisse prima della epistola $\mathrm{V}$, composta dopo il settembre del 1310, perché ideologicamente incompatibile con quella. Non discute questa testimonianza Pellegrini, Dante tra Romagna e Lombardia, che si concentra su quella relativa al primo soggiorno a Forlì (pp. 39-62). A beneficio di analisi future, converrà tenere separati i vari elementi della testimonianza di Biondo (esistenza dell'epistola, luogo di composizione, mittenti, destinatario e contenuto) data la possibilità che un nucleo originario fors'anche attendibile si mescolasse con spunti di provenienza disparata.

70 Davidsohn, Geschichte, III, p. 383 e 414; Davidsohn, Storia di Firenze, III, pp. 527-528; Bowsky, Henry VII, p. 36; Cardini, Cerchi, Giovanni. 
come la salutatio della prima epistola dove la «pars Alborum» è appunto quella «de Florentia», non un asse bianco provinciale. Lo prova parimenti la documentazione superstite relativa ad altri gruppi di esuli della stessa stagione politica. I lucchesi rifugiati a Pisa nel primo decennio del Trecento, ad esempio, «non cessarono mai di distinguersi dalla città che li aveva ospitati e continuarono a dirsi qui omnes sunt de Luca et modo habitatores pisane civitatis» ${ }^{71}$ e, quando nell'imminenza dell'arrivo di Enrico VII si mobilitano per ottenere l'agognato rientro in patria, si costituiscono nella «universitas exititiorum de Lucca»: a questo nome inviano due ambasciatori ai legati imperiali nel giugno del 1310 per giurare fedeltà al Lussemburghese. ${ }^{72}$ A loro volta, il 14 settembre 1313, quando i fuoriusciti fiorentini scrivono a Niccolò da Prato per discutere della sorte del domenicano accusato di avere avvelenato Enrico, si firmano «pars imperialis que est extra Florentiam». ${ }^{73}$ La definizione di Tuscia, di antica e illustre tradizione romana, vale all'inizio del XIV secolo quando la penisola si consideri dal punto di vista dell'imperatore o del pontefice; non è invece operativa dal punto di vista di lucchesi, pisani, pistoiesi, aretini, fiorentini e via dicendo che, anche quando estrinseci, continuano a mutuare la definizione delle loro identità dalle città di origine, e del resto l'impero sa perfettamente ricorrere alle categorie comunali correnti e nelle missive così come negli altri documenti pubblici, quando si tratta di extrinseci, si precisa sempre di quale città lo siano. ${ }^{74}$ Che nell'antropologia politica d'inizio Trecento esuli e fuoriusciti siano sempre nominati e sempre si nominino innanzitutto su scala municipale, non provinciale, non è del resto stupefacente: sono infatti prioritariamente cittadine le reti sociali all'interno delle quali, anche extra moenia, gli esuli continuano a muoversi, ed è normale che l'organizzazione e la mobilitazione politica avvenga su tale scala sia perché l'altra, che comporta la confederazione di esuli di provenienze diverse, è di gestione più complessa, per non dire impraticabile, per gruppi dall'alta mobilità e dalle risorse limitate, sia perché è appunto lo scacchiere

71 Cristiani, I fuorusciti toscani, p. 65.

72 Const. 4/2, no 1273, pp. 1407-1048. Si vedano anche l'instrumentum seguente $\mathrm{n}^{\circ} 1274$, pp. 1408-1409 con il quale l'«universitas exititiorum de Luca» nomina i propri capitani e il $\mathrm{n}^{\circ} 1286$, p. 1422 con cui appunta i propri legati e in cui si precisa che i membri della parte in questione sono «intus et extra civitatem» (del 7 ottobre 1311). Cfr. Cristiani, I fuoriusciti toscani, pp. 65-66, sulla scorta dello studio di Sforza, Castruccio Castracani, p. 29 e 46 (contenente un'edizione parziale del secondo documento), e Bowsky, Henry VII, p. 36.

73 Const. 4/2, n 1245, p. 1305.

74 A titolo di esempio cfr. Const. 4/2, $n^{\circ}$ 981, p. 1016 (su esuli vicentini e veronesi); $n^{\circ}$ 989, p. 1032 (sugli esuli bresciani); $n^{\circ}$ 1226, p. 1287 (sulla «pars extrinseca Bobii»); n 1227, p. 1289 (su quella di Lodi). Sulla definizione - implicitamente militare - di Tuscia, v. Faini, Italica gens, p. 140. 
cittadino il primo che interessa, dal momento che si tratta di negoziare innanzitutto il rientro in patria.

Rispetto a tale assetto, gli «omnes Tusci qui desiderant pacem» costituiscono dunque come un'anomalia discorsiva, che corrisponde più alla proiezione sullo scenario peninsulare della griglia provinciale che a una comunità politica empirica. Questa serie di riflessioni sconsiglia dunque, in assenza di documentazione storica di diverso segno, d'intendere la salutatio della VII epistola dantesca come la prova dell'esistenza di una qualche pars sovra-municipale di esuli radunati in Casentino a nome della quale e su committenza della quale Dante prendesse l'incarico d'indirizzarsi all'imperatore.

\section{Co-mittenti e destinatari multipli}

Si è osservato che la dicitura «omnes Tusci qui desiderant pacem» può difficilmente riferirsi, storicamente e retoricamente, alla o alle corti casentinesi dei conti Guidi o a una compagine di esuli toscani che, organizzata, avesse dato a Dante il ruolo di rappresentante ufficiale del gruppo. Che farne allora? La comprensione di questa formula, e più in generale, del gesto testuale della VII epistola esce da quest'analisi irrimediabilmente compromessa, l'una vuota e senza termini di paragone nella sua eccentricità, l'altro inspiegabile e muto se non più inquadrabile in una qualche catena di comando Guidi/fuoriuscitiDante-Enrico VII?

Per provare a rispondere e a ripensare questo testo in una prospettiva diversa occorre, in primo luogo, apprezzare la fattura tutta dantesca della definizione. Per giro sintattico e idiosincrasie lessicali gli «omnes Tusci qui pacem desiderant» ricalcano un modulo prediletto da Dante, specie in sede proemiale. Valga il raffronto con gli incipit del Convivio e della Monarchia nei quali si afferma l'universalità del desiderio di sapere, dal quale appunto discende la necessità dell'Impero universale. ${ }^{75}$ Non meno dantesco è, in questo giro d'idee, il richiamo alla pace. Altri hanno giustamente osservato l'importanza del tema, vero basso continuo di tutta la produzione epistolografica pro-arrighiana. ${ }^{76}$ Per

75 Conv. I, i, 1: «sì come dice lo Filosofo nel principio della Prima Filosofia, tutti li uomini naturalmente desiderano di sapere»; e ancor meglio Mon. I, i, 1: «omnium hominum, quos ad amorem veritatis natura superior impressit, hoc maxime interesse videtur, ut, quemadmodum de labore antiquorum ditati sunt, ita et ipsi posteris prolaborent, quatenus ab eis posteritas habeat quo ditetur». Per questo principio cardinale della riflessione dantesca, cfr. Falzone, Desiderio della scienza.

76 Ep. (Honess), p. 27. 
parte mia mi pare importante sottolineare un dato ovvio ma che tende talvolta a passare in secondo piano: che l'evocazione della missione pacificatrice dell'impero volge qui all'esortazione alla guerra, e addirittura alla guerra contro la patria dell'esortatore. Ciò interessa non solo perché la salutatio offre un altro capitolo alla lunga storia del topos della guerra giusta, ma perché ciò riconduce a un problema che è dei più centrali e ardui nella meditazione dantesca durante l'esilio: si consideri la serie di ripensamenti e aggiustamenti dal Farinata condannato per lo scempio dell'Arbia ma elogiato per il contegno ad Empoli di Inferno X, alla rilettura di Montaperti come exemplum di superbia punita in Purgatorio XI, passando per il sofferto presagio di catastrofe ultrice dell'incipit di Inferno XXVI, e ancora la spinosissima rivisitazione del personaggio lucaneo di Curione che qui offre la sua battuta a Dante (Ep. VII, 16) ma figura tra i seminatori di scismi e discordia in Inferno XXVIII, senza dimenticare l'ammissione di colpa della «montanina» (v. 81) e, prima ancora, le riflessioni che doveva contenere l'epistola perduta Popule mee. ${ }^{77}$ Inoltre, e come Dante spiega subito appresso nella VII lettera tramite il proverbium giovanneo e argomenta altrove più distesamente, ${ }^{78}$ dato che il desiderio della pace è distintivo dell'umanità e voluto da Dio affinché il genere umano compia il proprio destino terreno, ne discende che chi ne è privo è perciò stesso estraneo all'umanità e ribelle alla Provvidenza. In altri termini, la relativa restrittiva della salutatio annuncia quella disumanizzazione e diabolizzazione del nemico fiorentino che, dopo l'ultimo invito alla penitenza dell'epistola VI, questa lettera e ancor più la Commedia porteranno alle estreme conseguenze. A sigla di queste considerazioni, non casualmente sull'esser priva di pace si arresta la pesante ironia dantesca contro Firenze alla sua climax: «tu ricca, tu con pace e tu con senno». ${ }^{79}$ In sintesi, il tardus «omnes Tusci qui pacem desiderant» è un vero e proprio distillato di dantismo che, nel lessico e pensiero di Dante, si traduce in 'tutti i Toschi filo-

77 Per questa riflessione in Dante, cfr. Brilli, Firenze e il profeta, pp. 47-49, 52, 181-193 e 301302.

78 «Immensa Dei dilectione testante relicta nobis est pacis hereditas, ut in sua mira dulcedine militie nostre dura mitescerent et in usu eius patrie triumphantis gaudia mereremur», subito opposto all'«antiquus et implacabilis hostis» (Ep. VII, i, 2 con riferimento a Io. XIV, 27). Sulla pace come oggetto del desiderio naturale, conditio sine qua non del conseguimento della felicità terrena, e obiettivo della provvidenza che sovrintende la storia umana, si vedano il capitolo «speziale» del Conv. (IV, iv-v) e Mon. I, iv, 2-6; I, xi, 14.

79 Purg. VII, 137. Somaini, Il quadro politico ha argomentato in favore della datazione della composizione del VII del Purgatorio «tra il luglio e il settembre del 1310, quando ormai potevano darsi per certe le notizie sull'arrivo imminente in Italia di Enrico VII di Lussemburgo» (p. 96) apportando una messe di elementi indiziari che meritano di essere attentamente meditati. 
imperiali' (non 'ghibellini'), perché sostenitori dell'Impero quale Dante lo teorizza, baluardo della realizzazione del fine terreno dell'umanità, voluto da Dio e fustigatore di ogni diabolica ribellione.

In secondo luogo, conviene prendere maggiormente sul serio - ossia sforzarsi di auscultare invece di occultare - l'ostentata vaghezza di questa dicitura in sede storico-politica, così come dei non specificati «altri» cui Dante si riferisce nel corpo della lettera. Come si è osservato, infatti, mentre la definizione fa appello a una categoria che l'imperatore ben poteva comprendere e sicuramente gli stava a cuore quale parte imprescindibile del suo regno a venire - la Tuscia -, essa è di matrice teologico-politica: quella Tuscia anelante la pace e che, in quanto tale, non può non riconoscersi nella missione del «Titan (...) pacificus» (Ep. V, 3).

Ma a che pro accreditare tali co-mittenti (non committenti)? A questo proposito si possono sviluppare due ordini di considerazioni. Da un lato, si può suggerire che questa scelta risponda al deficit di auctoritas e legittimità del primo mittente, il fiorentino immeritatamente esule che non poteva vantare nessun titolo e nessun incarico che lo autorizzassero a indirizzarsi a nome proprio a chi era emanazione diretta del «fons pietatis» (Ep. V, 7) o, come detto altrove, del «fons universalis auctoritatis» (Mon. III, xv, 15). I vaghi co-mittenti vengono così a puntellare il primo, dalla legittimità traballante, mitigando la distanza abissale che separa Dante dal suo destinatario e, di conseguenza, lo scandalo costituito da questa lettera nelle convenzioni epistolografiche dell'epoca; uno scandalo, si ricorderà, che, in tempi di paternità ancora dibattuta, a Francesco Novati pareva tale da costituire una prova di autenticità. ${ }^{80}$ Dall'altro, si potrà osservare che la vaghezza è qui direttamente proporzionale al potenziale inclusivo della definizione, applicabile tanto ai Guidi quanto ai fuoriusciti e ad ogni

$\mathbf{8 0}$ «Si noti però che queste lettere [quelle incluse nelle artes dictaminis], le quali muovono in genere da circostanze di fatto vere, hanno sempre per mittenti o per recipienti dei magistrati o altre persone investite di pubblici uffici: tutta la gerarchia sociale è sottoposta a rassegna, dall'imperatore al notaio, dal pontefice al chierico; ma non mai, ch'io sappia, vi si mettono in scena individui privati. Or perché si sarebbe da qualche dettatore fatta un'eccezione per Dante? E questo in tempi a lui vicini, quando cioè la sua fama era certo inferiore di molto a quanto divenne più tardi? Se fosse stata di moda l'avvalersi del nome d'individui reali, celebri, quantunque non investiti di dignità ufficiali, perché non troveremmo noi lettere attribuite a Cino da Pistoia, a Brunetto Latini, ad Albertino Mussato, ad altri cotali?» (Novati, Le epistole, pp. 20-21). Sulla correttezza, oltre che della conclusione, della premessa della riflessione di Novati mi permetto di rinviare a Brilli, The Interplay, pp. 161-166. Per quanto riguarda la complessità della fonction auteur nei testi epistolografici medievali, oltre a Grévin, Rhétorique du pouvoir, pp. 65 e ss. e Delle Donne, Autori, redazioni (già cit. in Brilli, The interplay, p. 163, nota 40), si veda il bel saggio di Stella, Chi scrive le mie lettere?, uscito a ridosso della consegna di quel lavoro. 
altro soggetto politico toscano disposto a riconoscere e sposare la visione dantesca dell'Impero. E ciò diviene fondamentale quando si osservi che, non diversamente dall'epistola V sebbene su una scala diversa, la VII ha tutta la qualità della lettera circolare o, se si preferisce, del «manifesto». ${ }^{81}$ Altrimenti detto, è possibile se non probabile che sia stata concepita, redatta e diffusa in vista di destinatari, in realtà, multipli. Accanto a quello ufficiale ed esplicito, Enrico VII, dei destinatari impliciti che, convinti dagli argomenti che vi sono esposti nonché variamente solleticati dalle molte ragioni che rendevano desiderabile un ridimensionamento dello strapotere di Firenze, avrebbero potuto sottoscriverla, riconoscersi nella definizione appositamente aperta della salutatio, accorrere in supporto di Enrico e, perché no?, fare di Dante il loro portavoce ex post. Se questo suggerimento suona troppo ingegnoso conviene forse rileggere la scena delle retrouvailles con Beatrice nel Purgatorio. Precisamente il passo nel quale la donna, rispondendo agli angeli che la interrogano sulle ragioni della sua durezza contro il pellegrino, teorizza appunto una strategia discorsiva obliqua, ossia un discorso pubblico inteso a raggiungere destinatari secondi (là Dante pellegrino, qui i potenziali sostenitori di Enrico) oltre ai manifesti (là gli angeli, qui l'imperatore): «voi vigilate ne l'etterno die, / sì che notte né sonno a voi non fura / passo che faccia il secol per sue vie; / onde la mia risposta è con più cura / che m'intenda colui che di là piagne, / perché sia colpa e duol d'una misura» (Purg. XXX, 103-108). Parlare (o scrivere), insomma, all'uno affinché l'altro intenda e sia riformato dal discorso così inteso.

Quando si legga la VII epistola come una missiva rivolta non solo all'imperatore ma anche ai suoi sostenitori potenziali, diviene tanto più assennata la denuncia della politica fiorentina che, approfittando dell'indugio imperiale, ingrassa le sue fila cooptando e irretendo nuovi sostenitori (Ep. VII, 15 e 23-28), così come l'insistenza sui motivi dell'esilio babilonese e dell'anelata reformatio e restitutio in favore delle vittime di precedenti ingiustizie (Ep. VII, 4 e 30). La prima doveva suonare come un campanello d'allarme alle orecchie di dinastie territoriali che, come i Guidi, avevano tutto l'interesse di non vedere rafforzato oltre il potere di Firenze e dunque di sostenere la campagna di Enrico VII; la seconda blandiva la sofferenza, la nostalgia e il desiderio di riscossa dei fuoriusciti della stagione politica precedente motivandoli parimenti a supportare la missione arrighiana. Se il Curione lucaneo, qui citato da Dante, spronava Cesare «dum trepidant nullo firmate robore partes, tolle moras» (Phars. I, 280-281, cit. in $E p$. VII, 16), quando quest'esortazione sia formulata pubblicamente, essa costituisce di per sé un invito a che le forze in campo si confermino e rinsaldino nella comune adesione al fronte imperiale.

81 L'espressione è di Mazzoni, Dante e la terra casentinese, p. V (nella rist. anastatica p. 103). 
Da questo punto di vista la VII epistola si pone in profonda continuità ideologica con le due precedenti, e in particolare con la V; se ne distanzia tuttavia per lo specificarsi dello scacchiere geo-politico, ora identificato con la Tuscia. Questo nuovo focus discende non solo da un maggiore, e del tutto comprensibile, interesse di Dante per i fatti di Toscana bensì anche da una corretta analisi dello scenario coevo e da un orizzonte d'attesa in sé tutt'altro che irragionevole. Da un lato, Dante certamente «ben vede, con la saggezza d'uomo politico che non possiamo disconoscere, che solo Firenze è il ganglio strategico della resistenza, il caposaldo da battere e annientare», ${ }^{82}$ ma anche comprende che, per avere una qualche possibilità di riuscita, è necessario innescare una cooperazione virtuosa tra forze imperiali e forze locali laddove queste ultime, come l'epistola lascia intendere e i fatti seguenti confermano, si sarebbero mobilitate solo all'arrivo dell'imperatore, non prima. Dall'altro, memore dell'investimento di alcuni Guidi nella stagione politica precedente, consapevole dei loro interessi nella regione e forte della consuetudine passata, recandosi «sub fonte Sarni»e lì componendo e diffondendo il suo manifesto, Dante ritiene di poter contribuire attivamente a che ciò avvenga. ${ }^{83}$

Questa spiegazione mi pare convenire meglio allo scenario del Casentino della primavera del 1311, uno scenario ben più opaco e fluido in termini di schieramenti politici di quel che postula l'esistenza di committenti di cui non restano tracce. Su questo scenario, Dante, forse anche non da solo - ma sia chiaro che una cosa è parlare magari tra amici delle notizie del giorno e discutere del da farsi, altro essere appuntato come portavoce ufficiale di questi o di quelli -, pensa di poter agire tramite la sua attività epistolografica, in particolare mettendo in moto un circolo virtuoso che avrebbe dovuto vedere Enrico VII scendere fulmineo contro Firenze e, congiuntamente, dall'Appennino e dalle altre aree toscane avanzare gli alleati pronti a dargli man forte. Come andò la storia è noto: neanche i Guidi riescono a mettersi d'accordo sulla linea da seguire e l'appello di Dante rimane senza seguito, facendo presa al massimo sulla Gherardesca che gli commissiona, nel maggio seguente, la redazione di una o più

82 Così Mazzoni, Le epistole, p. 75, con metafora organicistica che varia le dantesche dell' «hydra pestiferat» e della radice ( $E p$. VII, 20-21). Per la correttezza storica dell'analisi cfr. Bowsky, Florence and Henry e Bowsky, Henry VII in Italy.

83 In questa chiave, le riflessioni svolte da altri circa l'opportunità politica della VII lettera appaiono condivisibili, a patto però di non farne le prove di una committenza cui ostano le considerazioni svolte sopra, e di scorgervi invece le ragioni che fecero ritenere a Dante che il suo appello potesse far presa ed essere sottoscritto in primis, ma non esclusivamente, da interlocutori casentinesi, sorta di manifesto grazie al quale rassembrare le forze disperse sotto l'egida non di una parte bianca bensì dell' «uccel di Dio» (Par. VI, 4) e che, qualora accolto, lo avrebbe anche confortato nel suo ruolo, nient'affatto secondario, di cantore dell'Impero. 
lettere in risposta (si badi) all'imperatrice, certo utili se l'imperatore e la sua corte fossero arrivati un giorno da quelle parti. Dall'esito, sotto questo profilo, fallimentare dell'appello non si deve tuttavia desumerne l'assurdità nel momento in cui è formulato; e neanche il fallimento in sé deve essere eccessivamente enfatizzato: se l'epistola VII non riesce a far evolvere la scacchiera come l'autore ha sperato, è però anche grazie a questa pubblica militanza, oltre che alla produzione letteraria, che Dante riesce a procacciarsi il favore e l'ospitalità del signore di Verona quando la situazione politica volge al peggio. Un esito questo, certo, infinitamente inferiore all'auspicato ma non per questo irrisorio.

\section{Epistolografia performativa e profetismo}

Ho premesso che quest'analisi avrebbe avuto conseguenze anche sul modo d'interpretare il gesto testuale di Dante, e occorre svolgerle. Le ragioni storiche e retoriche esposte sopra invitato a ritenere che la VII epistola non possa leggersi come un documento emanante da una comunità politica empirica già definita e storicamente operante al momento della sua redazione, che avesse incaricato Dante di rappresentarla per iscritto. Al contrario, questa lettera può e personalmente ritengo che debba essere letta come un tentativo discorsivo volto a fondare una comunità politica possibile: una comunità che ancora non è ma che, a fronte delle manovre fiorentine e con l'avvento di Enrico VII in Toscana, avrebbe potuto mettersi in opera. I co-mittenti definiti nella salutatio come «omnes Tusci qui pacem desiderant» si situano allora non a monte del discorso epistolografico, quali dei committenti propriamente intesi, bensì dentro di esso, perché costruiti dalla lettera, e ottativamente a valle, perché la lettera ambisce a tradurli in realtà fuori di sé, quali suo effetto.

Osservare ciò equivale a considerare quello sviluppato nella VII epistola come un discorso performativo nel senso austiniano del termine, un discorso cioè che non descrive la realtà bensì intende, col suo darsi, agirla e modificarla. Più in particolare, si tratta di un discorso di tipo "perlocutorio», dal momento che l'esito dipende dalla reazione dei destinatari - tanto l'esplicito, Enrico VII, quanto gli impliciti, i toschi che se avessero amato la pace (e soprattutto se l'avessero intesa come Dante la intendeva) avrebbero dovuto caldeggiare l'avvento dell'imperatore e supportarne l'azione contro Firenze. ${ }^{84}$ L'interesse di

84 Il riferimento è ovviamente a Austin, How to Do Things. Per un'interessante applicazione delle categorie austiniane alle teorie del linguaggio scolastico, cfr. Rosier Catach, La parole efficace. 
questo suggerimento risiede meno nell'apporre un'etichetta che nelle sue conseguenze sulla valutazione dell'iter di Dante autore, sia nel suo complesso sia in relazione al problema specifico del profetismo. Rispetto al complesso di quest'itinerario, la concezione performativa che Dante ha dello scrivere e della propria attività letteraria sta emergendo sempre con maggiore finezza negli studi più recenti, e in relazione già alla Vita Nova: da questo punto di vista, dunque, il Dante mobilitato nella campagna filo-arrighiana sta semplicemente esportando a un discorso pubblico, ossia di pubblicistica, delle modalità compositive e più in generale un approccio alla composizione che aveva già sperimentato, senza dubbio con successo, in altri ambiti. ${ }^{85}$ "Semplicemente", s'intende, da un punto di vista operazionale, perché invece il risultato del trasferimento da testi pur sempre di fictio a un discorso pubblico di altra natura è retoricamente dirompente.

Con ciò s'arriva all'apporto specifico di questa ricognizione in vista del profetismo o, per dir meglio, dell'assunzione da parte di Dante di una postura di stampo profetico. ${ }^{86}$ Come altri studiosi in passato, si pensi a Bruno Nardi e Nicolò Mineo, sono portata a riconoscere nelle epistole della stagione arrighiana un punto di svolta nella produzione dantesca rispetto a tale questione; e altrove, dialogando con chi insiste sull'appartenenza di questi testi al genere del dictamen medievale per disattivarne la carica profetica, ho ricordato che questa lettura, che pure muove da dati formali e stilistici oggettivi e condivisibili, non può però arrivare al punto di obliterare un dato testuale altrettanto lampante: il fatto che la più grande infrazione dantesca alle consuetudini epistolografiche del suo tempo, e pertanto ciò che vi è di retoricamente "profetico", è la sovraesposizione della voce e della persona autoriale, la messa in scena cioè di sé, fin dalle salutationes, quale interlocutore privilegiato dei grandi della terra, dei fiorentini, dell'imperatore stesso. ${ }^{87}$ Continuando a ragionare in questa prospettiva, gli «omnes Tusci qui pacem desiderant» e la disperata impresa performativa della VII epistola spiegano come ciò sia potuto accadere.

Lungi dall'essere un discorso su commissione o un discorso visionario, il dantesco è un tentativo di fondare, in una congiuntura storica ancora relativa-

85 Penso ad esempio ai lavori di Gragnolati, Authorship and Performance e Gragnolati, Una performance senza gerarchia, che ringrazio l'autore per aver condiviso in anteprima, sulla Vita Nova, che integrando la categoria austiniana offrono una lettura del libello come opera di costruzione attraverso la riscrittura delle liriche precedenti.

$86 \mathrm{Nel}$ senso con cui il termine è adottato da Piron, La parole prophétique.

87 Nardi, Dante profeta, p. 339; Mineo, Mondo classico, p. 71; Brilli, Firenze e il profeta, pp. 358 e ss. (con bibliografia pregressa); per le considerazioni qui richiamate si veda in particolare Brilli, The interplay, pp. 161-169. Diversamente, come si ricorderà, per Morghen il punto di svolta era costituito dall'epistola XI (Morghen, Dante profeta, p. 1152). 
mente aperta, una comunità politica a venire tramite la parola, in particolare esportando all'ambito dell'epistolografia politica le modalità di costruzione e auto-accreditazione del sé, così come di costruzione del pubblico, che Dante aveva già messo in opera con frutto in altra sede. In altri termini, la messa in scena di sé nelle epistole serve affinché altri seguano e sottoscrivano; ma, al tempo stesso, ciò stravolge il genere perché v'immette un dispositivo a esso estraneo e che, incentrato sull'auto-promozione di un singolo in quanto portavoce di una verità che lo trascende, s'apparenta al profetismo. Si vede allora bene come l'habitus profetico dantesco (non parlo qui delle sue intime convinzioni, che sono il terreno della psicologia storica o della divinazione) sia andato definendosi per scarti sia pure radicali e determinati dall'incalzare degli eventi ma che, per altro verso, si collocano su un'ideale linea di continuità con le prove della giovinezza e con quella fideistica convinzione nel potere della (propria) parola. Anche s'intravvede perché, dopo aver cercato di fondare in tale modo una nuova comunità e quando capitò che nessuno seguisse, il sé così sovraesposto e quasi nudo si risolvesse a reclamare il proprio fare «parte per sé stesso», accreditando un'investitura ora apertamente divina e perciò incontrovertibile, e contemporaneamente dedicandosi totalmente alla costruzione, stavolta pienamente riuscita, di un'altra comunità: quella di «coloro / che questo tempo chiameranno antico» (Par. XVII, 119-120), quella di noi che ancora oggi lo leggiamo e ne discutiamo. 
